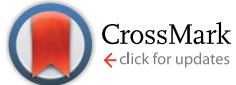

Cite this: RSC Adv., 2015, 5, 18075

Received 1st January 2015

Accepted 4th February 2015

DOI: $10.1039 / \mathrm{c} 5 \mathrm{ra00015g}$

www.rsc.org/advances

\title{
Functionalization of photochromic dithienylmaleimides $\dagger$
}

\author{
D. Wutz, C. Falenczyk, N. Kuzmanovic and B. König*
}

Photochromic dithienylmaleimides are well known molecular switches, but for applications the suitable functionalization of the photochromic scaffold is required. We report here synthetic routes to dithienylmaleimides, which are functionalized at three different positions: at each of the thiophene moieties and the maleimide nitrogen. A Perkin-type condensation of two thiophene precursors is used as the key step to assemble the maleimide core, which allows the synthesis of non-symmetrically substituted dithienylmaleimides, such as photochromic amino acids. A different approach to the maleimide core is provided by the reaction of a dithienylmaleic anhydride with amines or hydrazides leading to maleimide protected dithienylmaleimides and photochromic labeled natural amino acids. The photochromic properties of the new photoswitches were investigated showing reversible photochromism in polar organic solvents.

\section{Introduction}

Photochromism has attracted great attention in materials science $^{1}$ and as a tool in molecular biology. ${ }^{2} \mathrm{~A}$ variety of applications are found in molecular optoelectronics ${ }^{3}$ and optical data storage. ${ }^{4-6}$ In the field of life sciences, molecular switches have been used to control enzyme activity, ${ }^{7-10}$ Watson-Crick base pairing, ${ }^{11-13}$ the regulation of neuronal activity by photochromic ligands for ion channels and receptors, ${ }^{14-20}$ antibiotic effects ${ }^{21,22}$ and even the agility of a living organism ${ }^{23}$ by light. This broad applicability is one of the reasons why photopharmacology has evolved into a vibrant field of research..$^{24}$ Various photochromic molecules, like azobenzenes ${ }^{25}$ spiropyrans, ${ }^{26}$ spirooxazines, ${ }^{26}$ fulgides $^{27}$ and diarylethenes ${ }^{28,29}$ have been developed. All these photoswitches can be reversible toggled between two isomers using light. The well investigated dithienylethenes (DTEs), including dithienylmaleimides, are characterized by a nearly quantitative photochemical conversion between the photoisomers, which are often thermally stable. Irradiation with light of a specific wavelength switches the DTEs between their open and closed photoisomers, which differ in conformational flexibility and electronic conjugation (Fig. 1).

Many DTEs show high fatigue resistance. ${ }^{28}$ Despite their outstanding photophysical properties the synthesis of DTEs, in particular of non-symmetric derivatives, is laborious..$^{13,30}$ Different synthetic routes for the preparation of dithienylmaleimides were established. Starting from 3,4-

Institute of Organic Chemistry, University of Regensburg, D-93051 Regensburg, Germany.E-mail: burkhard.koenig@ur.de

$\dagger$ Electronic supplementary information (ESI) available: Additional experimental data and ${ }^{1} \mathrm{H}$ and ${ }^{13} \mathrm{C}-\mathrm{NMR}$ spectra of all prepared compounds. See DOI: 10.1039/c5ra00015g dibromomaleimides and 3,4-diiodomaleimides, respectively, both thiophene moieties can be attached by palladium catalyzed Suzuki coupling. ${ }^{31-33}$ However, only nitrogen protected maleimides can be used and the synthesis of non-symmetric compounds is challenging. Another route uses the reaction of a dithienylmaleic anhydride with amines to the corresponding maleimide. ${ }^{34-36}$ The synthesis of diarylmaleimides by intramolecular Perkin condensation of two independently prepared precursors gives selective access to non-symmetric diaryImaleimides. ${ }^{10,37,38}$ Compared to diarylperfluorocyclopentenes and diarylcyclopentenes, diarylmaleimides are more hydrophilic and better water soluble, which is valuable for applications in biology and pharmacy. The absorption maxima of diarylmaleimides are shifted to higher wavelengths and thus the photoisomerization can be induced by light with lower energy reducing potential cell damage. ${ }^{28}$ Moreover, the biocompatibility of diarylmaleimides is known from bisindolylmaleimides, for instance arcyriarubins and arcyriaflavins with antibiotic activities, several other potent protein kinase
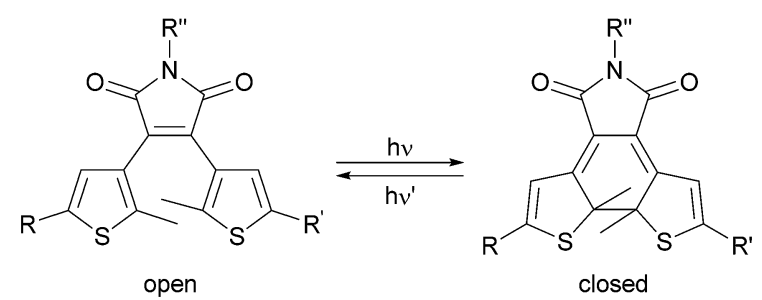

Fig. 1 Reversible photochemical isomerization of a dithienylmaleimide between the open and closed photoisomer by irradiation with light of different wavelength. 
and sirtuin inhibitors. ${ }^{\mathbf{1 0 , 3 9 - 4 3}}$ However, a better synthetic access to functionalized photochromic dithienylmaleimides is desirable in order to extend their applications. Herein we discuss the synthesis of functionalized dithienylmaleimides substituted on each thiophene moiety and the maleimide nitrogen atom.

\section{Results and discussion}

\section{Synthesis}

Functionalization of the maleimide nitrogen atom. The transformation of diarylmaleic anhydrides into their corresponding diarylmaleimides provides an easy access to compounds with a functionalized maleimide nitrogen atom. ${ }^{28}$ Complex functionalities or protecting groups can be introduced at the maleimide nitrogen by reaction with amines or hydrazides. We used the adapted synthetic approach of Scandola et $a l .{ }^{36}$ for the synthesis of anhydride $\mathbf{4}$ as precursor (Scheme 1).

Methyl ester 2 was converted to its potassium salt $\mathbf{3}$ and condensed in a Perkin reaction with carboxylic acid $\mathbf{1}$ yielding
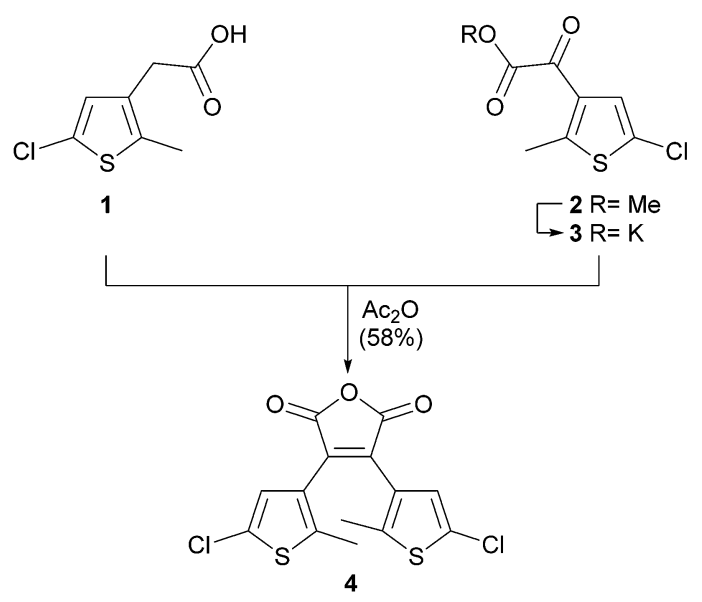

Scheme 1 Synthesis of dithienylmaleic anhydride 4 . the photochromic maleic anhydride 4. The anhydride moiety allows the subsequent functionalization with hydrazides or amines (Scheme 2). Therefore maleic anhydride 4 was treated with $\alpha$-Cbz protected L-glutamic acid $\gamma$-hydrazide ${ }^{44}(5)$ and $\alpha$ $\mathrm{Cbz}$ protected L-lysine to give amino acids 6 and 7 with a photochromic dithienylmaleimide on each sidechain. Photochromic tripeptides forming hydrogels with different aggregation modes mainly depending on the switch moiety were recently reported..$^{45}$ The reaction of hydrazine hydrate in acetic acid as solvent and 1,2-dimethylhydrazine dihydrochloride, respectively, with maleic anhydride 4 afforded the maleimide nitrogen protected dithienylmaleimides $\mathbf{8}$ and $\mathbf{9}$ in good yields (Scheme 2). Remarkably, the formation of any maleic hydrazide or other tautomers was not observed. The protected maleimides 8 and $\mathbf{9}$ could be used for further functionalizations on the thiophene moieties by palladium-catalyzed cross coupling reactions or other reactions using the reactivity of the heteroaryl chlorides.

Functionalization as photochromic amino acid. Recently, DTE-based non-natural amino acids were synthesized and successfully introduced into small peptides. ${ }^{46}$ However, their water-solubility is limited due to the diaryl perfluorocyclopentene core and therefore we developed a more polar dithienylmaleimide amino acid. Compounds 13a and 13b were prepared by a Perkin condensation ${ }^{10,37,38}$ of the thiophene precursors $\mathbf{1 0}$ and $\mathbf{1 1}$ bearing a protected primary amino or carboxyl group, respectively (Scheme 3). The Alloc group was chosen as a suitable protection for the amine as it is stable during the synthesis of compound 12. Diester thiophene 11 provides in 4-position the carboxylic ester giving the maleimide core in the Perkin condensation. The ester in 2-position will serve as carboxylate of the amino acid. Both carboxylic acids were protected as methyl ester. Alloc group and methyl ester of 12 were cleaved simultaneously with boron tribromide giving amino acid 13a in $47 \%$ yield, accompanied by $20 \%$ of the Alloc<smiles>CC(=O)NN1C(=O)C(c2cc(Cl)sc2C)=C(c2cc(Cl)sc2C)C1=O</smiles>

Scheme 2 Synthesis of the functionalized photochromic dithienylmaleimides 6-9 starting from maleic anhydride 4. 


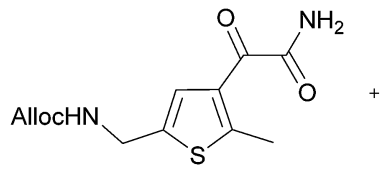

10<smiles>CCCCCCNCc1cc(C2=C(c3cc(C(=O)OC)sc3C)C(=O)NC2=O)c(C)s1</smiles><smiles>[R]NCc1cc(C2=C(c3cc(C(=O)O)sc3C)C(=O)NC2=O)c(C)s1</smiles>

Scheme 3 Perkin condensation of thiophenes 10 and 11 yielding dithienylmaleimide 12 and after deprotection 13a and 13b.

amino acid $\mathbf{1 3 b}$ as byproduct. A selective non-hydrolytic deprotection of the methyl ester of $\mathbf{1 2}$ is possible in low yield using lithium iodide in a polar aprotic solvent. ${ }^{47,48}$ A large excess of lithium iodide and reflux were necessary to achieve conversion; several solvents were tested with best yields in acetone (see ESI, Table S1†). Standard basic hydrolytic conditions for the deprotection of the methyl ester afforded the deprotected maleic anhydride (see ESI, Scheme $\mathrm{S} 2 \dagger$ ). The synthesis of thiophene 10 is depicted in Scheme 4 . Bromination ${ }^{49}$ of 2-methylthiophene (14) and subsequent Rosenmund-von Braun reaction $^{50}$ giving nitrile 16 were performed according to literature procedures.

The reduction of nitrile $\mathbf{1 6}$ with lithium aluminum hydride followed by immediate protection with allyl chloroformate afforded carbamate $\mathbf{1 7}$ in good yield. Using Fmoc chloride instead led to the respective Fmoc derivative in lower yields and caused the formation of side products in the subsequent Friedel-Crafts acylation. The yield of glyoxylester 18 in the Friedel-Crafts acylation depends critically on the sequence of the reagent addition. Best results were obtained by mixing 17 and methyl chlorooxoacetate before adding aluminum chloride in small portions. Quenching the reaction with saturated sodium hydrogen carbonate solution avoids the addition of hydrochloric acid to the allyl double bond. Aminolysis with aqueous ammonia converted the glyoxylester 18 in high yield
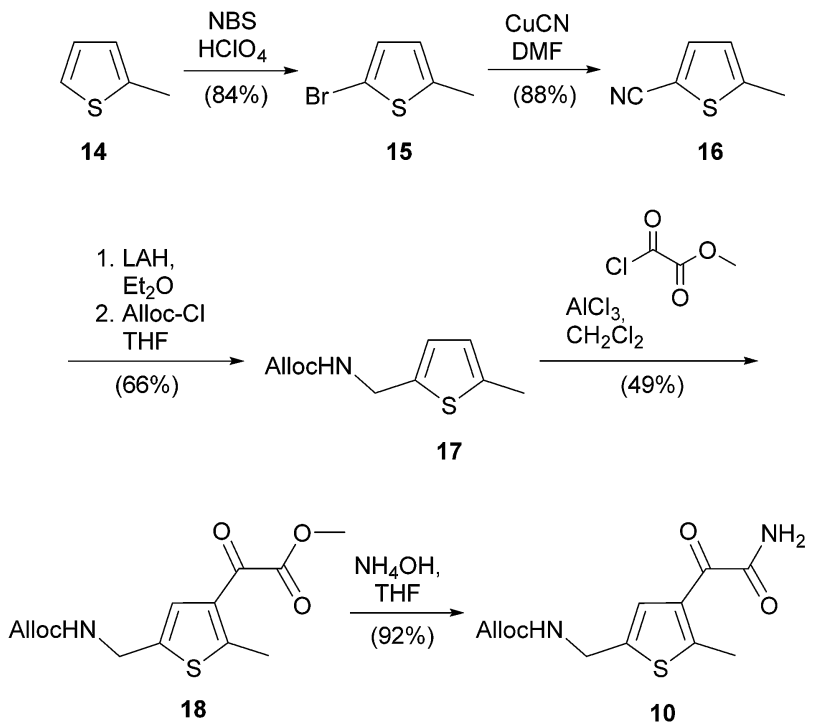

Scheme 4 Synthesis of thiophene 10

into compound 10. The overall yield for $\mathbf{1 0}$ after six steps is $22 \%$.

Thiophene $\mathbf{1 1}$ was prepared by esterification ${ }^{51}$ of methylthiophene acid 19 in the presence of thionyl chloride followed by Friedel-Crafts acylation and finally a thallium trinitrate (TTN) mediated oxidative rearrangement ${ }^{52}$ (Scheme 5). All intermediates were isolated in good to excellent yields with an overall yield of $68 \%$ for three steps. Initial moderate yields for the Friedel-Crafts acylation of around $40 \%$ significantly increased to $77 \%$ after rigorous removal of stabilizers from the solvent chloroform.

Functionalization by Suzuki coupling. Dithienylmaleimides are conveniently synthesized by the Perkin-type condensation. The reaction of two precursors yields the maleimide core without the need for protection of the maleimide nitrogen. Scheme 6 shows the intramolecular Perkin condensation of the
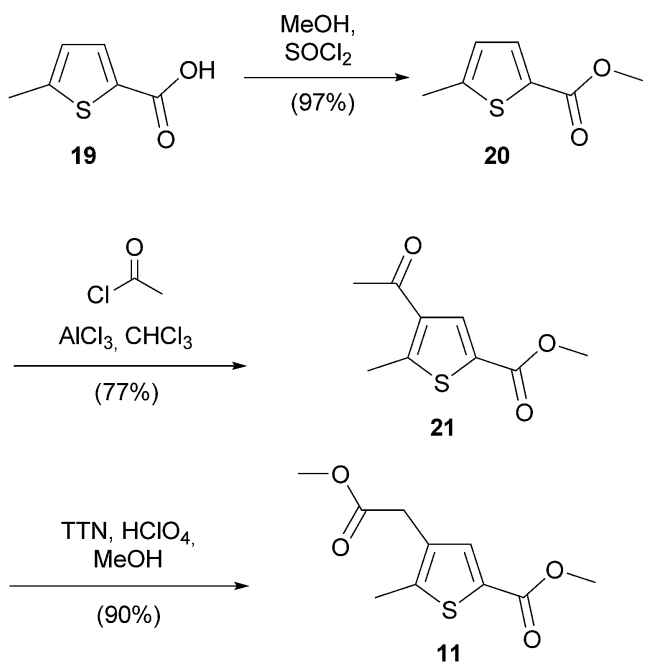

Scheme 5 Synthesis of thiophene 11. 


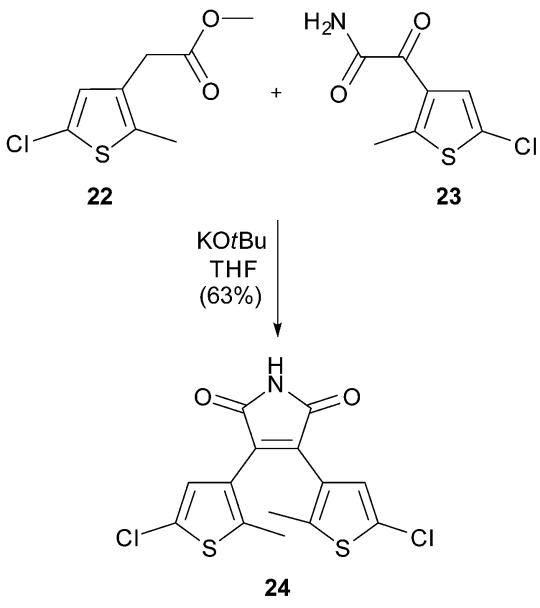

Scheme 6 Perkin condensation of 22 and 23 yielding dithienylmaleimide 24 .

two chlorosubstituted precursors 22 and 23. Both precursors can be differently functionalized by Suzuki coupling before used in the Perkin condensation yielding non-symmetric dithienylmaleimides.

Recently, we described the synthesis of symmetric diarylmaleimides, with thiophene moieties functionalized by palladium-catalysis prior to the condensation reaction. ${ }^{\mathbf{1 0}}$ Based on this strategy we prepared a small series of non-symmetric diarylmaleimides (Scheme 7).

The Perkin condensation to the maleimide core was performed under basic conditions combining the different
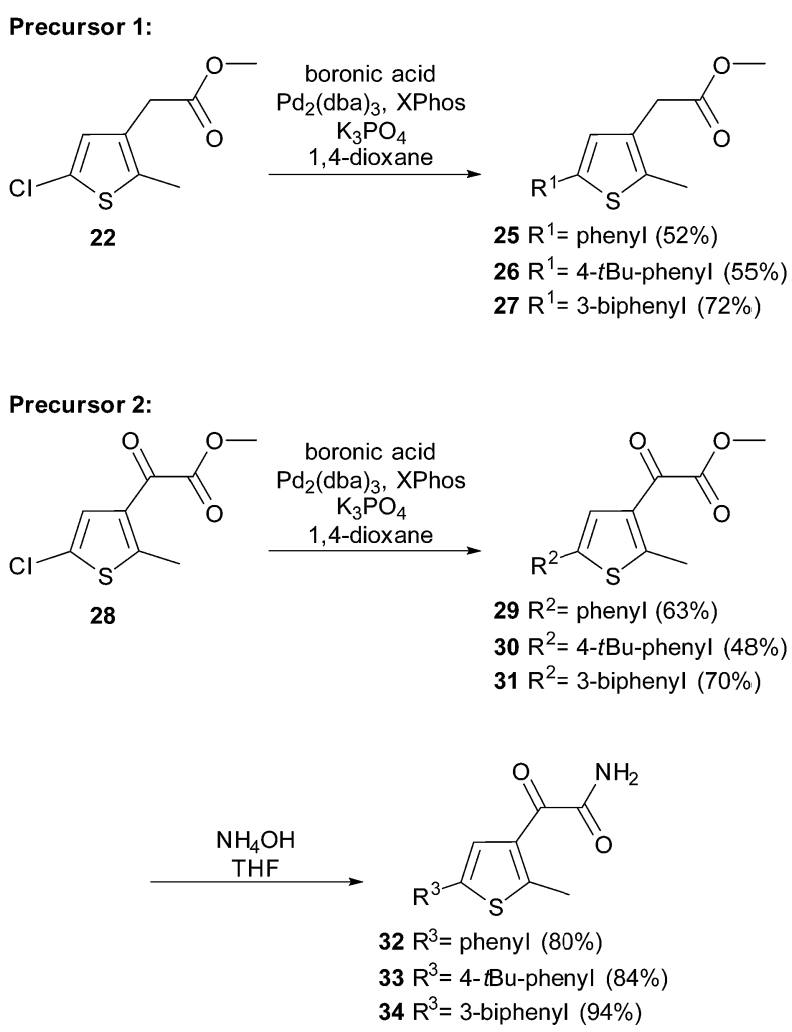

Scheme 7 Synthesis of functionalized precursors 25-27 and 32-34.

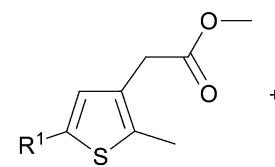

$26 R^{1}=4-t$ Bu-phenyl $27 R^{1}=3$-biphenyl<smiles>[R]c1cc(C(=O)C(N)=O)c(C)s1</smiles>

$23 \mathrm{R}^{2}=\mathrm{Cl}$ $33 R^{2}=4-t$ Bu-phenyl

$$
\begin{aligned}
& 35 \mathrm{R}^{1}=4-t \mathrm{Bu}-\text { phenyl, } \mathrm{R}^{2}=\mathrm{Cl}(28 \%) \\
& 36 \mathrm{R}^{1}=3 \text {-biphenyl, } \mathrm{R}^{2}=\mathrm{Cl}(27 \%) \\
& 37 \mathrm{R}^{1}=3 \text {-biphenyl, } \mathrm{R}^{2}=4-\mathrm{B} \mathrm{B} \text {-phenyl (33\%) }
\end{aligned}
$$

Scheme 8 Synthesis of non-symmetric substituted dithienylethenes 35-37 by Perkin condensation.

thiophenes. Scheme 8 summarizes the synthesis of the nonsymmetric photoswitches 35-37.

Photochromic properties. The dithienylmaleimide core structure can be toggled reversibly between a ring-open and ring-closed photoisomer (Fig. 1). The photochemical properties of photochromic compounds 4, 6-9, 12, 13a, 13b, 24 and 35-37 were investigated by UV-Vis spectroscopy. Despite of reports that diarylmaleimides are not able to undergo photoisomerization in polar solvents due to a twisted intramolecular charge transfer (TICT), ${ }^{53-55}$ we could observe reversible photoisomerization of the dithienylmaleimides 6-9, 12, 13a, 13b, 24 and 35-37 in methanol or dimethylsulfoxide, respectively. Fig. 2 shows the changes of the UV-Vis spectra of compound 12 upon irradiation with light of $312 \mathrm{~nm}$ (Herolab, $6 \mathrm{~W}$ ).

Upon irradiating a methanol solution of the ring-open form of compound 12 with UV light (312 nm), the absorption band at

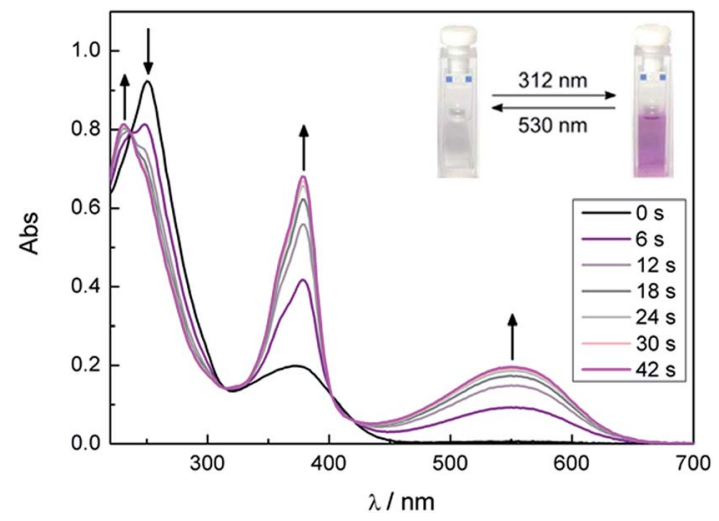

Fig. 2 Changes of the UV-Vis absorption spectra of dithienylmaleimide amino acid $12(50 \mu \mathrm{M}$ in $\mathrm{MeOH})$ upon light irradiation with $312 \mathrm{~nm}$; arrows indicate the changes of the absorption maxima over $42 \mathrm{~s}$ irradiation in periods of $6 \mathrm{~s}$ (Herolab, $6 \mathrm{~W}$ ); the cuvettes show the color of the solution before and after irradiation. 


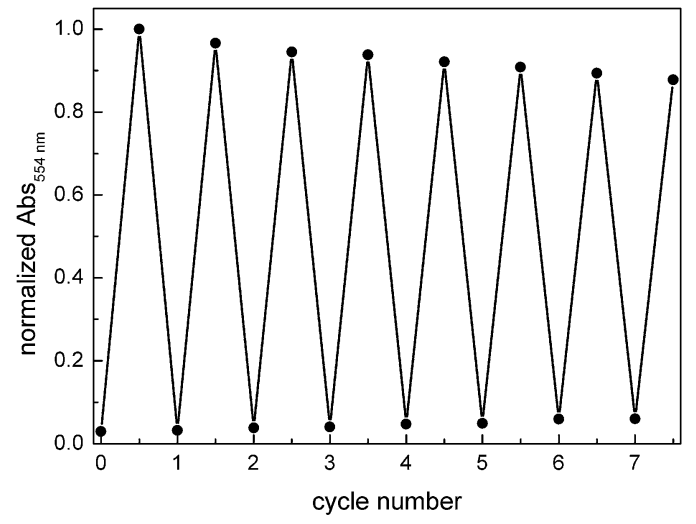

Fig. 3 Cycle performance of the dithienylmaleimide amino acid 12 $(50 \mu \mathrm{M}$ in $\mathrm{MeOH})$. Changes in absorption at $554 \mathrm{~nm}$ were measured during alternate irradiation with light of $312 \mathrm{~nm}$ for $60 \mathrm{~s}$ (Herolab, $6 \mathrm{~W}$ ) and $530 \mathrm{~nm}$ (CREE-XP green, $700 \mathrm{~mA}$ ) for $5 \mathrm{~min}$.

$250 \mathrm{~nm}$ immediately decreases. Simultaneously, new absorption maxima at $232 \mathrm{~nm}, 378 \mathrm{~nm}$ and $550 \mathrm{~nm}$ arise (Fig. 2) causing the color change of the sample from slightly yellow to purple. The isosbestic points indicate a clean conversion between two components. Compared to typical DTEcyclopentenes the absorption maxima are red shifted. The photostationary state was reached after $42 \mathrm{~s}$ of irradiation (Herolab, $312 \mathrm{~nm}, 6 \mathrm{~W}$ ) and the open form can be regained by irradiation with visible light $(>420 \mathrm{~nm}$ ) for $5 \mathrm{~min}$. The photoswitchable amino acid $\mathbf{1 2}$ is stable over at least seven closing/ opening cycles (Fig. 3).

The absorption maxima and their corresponding extinction coefficients for the open and closed form of all synthesized photochromic compounds are summarized in Table 1. Interestingly, the long wavelength absorption maximum of compound 13a is blue shifted to $537 \mathrm{~nm}$ compared to photoswitches 12 and 13b, which may indicate an interaction of the Alloc group with the dithienylmaleimide core. In contrast the selective removal of the methyl ester has almost no influence on the photochromic properties. In comparison to bischloro dithienylmaleimide 24 the functionalized maleimides $35-37$ show a bathochromic shift in their absorption maxima of the closed photoisomer. The enlarged $\pi$-system of the substituted thiophenes can explain this shift to higher wavelengths.

\section{Conclusions}

In summary, we have prepared several photochromic dithienylmaleimides. Maleimide nitrogen atom functionalized derivatives were obtained by the reaction of dithienylmaleic anhydride with different hydrazides and amines. Using a Perkin-type condensation non-symmetric dithienylmaleimides were synthesized including a photochromic amino acid and dithienylmaleimides with different aromatic substituents on each thiophene moiety. Reversible photoisomerization in dimethylsulfoxide and methanol was observed for all synthesized photochromic compounds.

\section{Experimental section}

\section{General information}

Commercial reagents and starting materials were purchased from Acros Organics, Alpha-Aesar, Fluka, Sigma Aldrich or VWR and used without further purification. Solvents were used in p.a. quality and dried according to common procedures, if necessary. To purify the chloroform for Friedel-Crafts acylations, it was washed with sulfuric acid $(1 \mathrm{M})$, dried over calcium chloride, filtered through silica, subsequently refluxed with phosphorus pentoxide $\left(5-10 \mathrm{~g} \mathrm{~L}^{-1}\right)$ and distilled under nitrogen atmosphere. Compounds $1,{ }^{10} 2,{ }^{10} 5,{ }^{44} 15,{ }^{49} 16,{ }^{50} 20,{ }^{51} 22,{ }^{10} 25,{ }^{10}$ $28,{ }^{10} 29^{10}$ and $32{ }^{10}$ were prepared according to previously reported procedures. Flash column chromatography was performed on a Biotage Isolera One automated flash purification system with UV/Vis detector using Sigma Aldrich MN silica gel $60 \mathrm{M}(40-63 \mu \mathrm{m}, 230-400 \mathrm{mesh})$ for normal phase or pre-packed Biotage SNAP cartridges (KP-C18-HS) for reversed phase chromatography. Reaction monitoring via TLC was performed on alumina plates coated with silica gel (Merck silica gel $60 \mathrm{~F}_{254}$, $0.2 \mathrm{~mm}$ ). Melting points were determined using a Stanford

Table 1 UV-Vis spectroscopic data of the open and closed (PSS) form of the synthesized photochromic compounds ${ }^{a}$

\begin{tabular}{llcll}
\hline Compound & Solvent & Conc. $[\mu \mathrm{M}]$ & $\lambda_{\max }$ open $(\varepsilon)$ & $\lambda_{\max }$ closed $(\varepsilon)$ \\
\hline $\mathbf{4}$ & $\mathrm{MeOH}$ & 100 & $242(26.0), 298(9.5)$ & $359(16.0), 523(3.7)$ \\
$\mathbf{6}$ & $\mathrm{DMSO}$ & 50 & $387(4.6)$ & $359(17.4), 510(3.2)$ \\
$\mathbf{7}$ & $\mathrm{DMSO}$ & 50 & $381(5.0)$ & $355(20.8), 500(4.1)$ \\
$\mathbf{8}$ & $\mathrm{DMSO}$ & 50 & $386(6.0)$ & $359(22.7), 508(4.3)$ \\
$\mathbf{9}$ & $\mathrm{DMSO}$ & 50 & $380(3.3)$ & $351(12.9), 498(2.7)$ \\
$\mathbf{1 2}$ & $\mathrm{MeOH}$ & 50 & $250(18.5)$ & $232(16.2), 378(13.6), 550(3.9)$ \\
$\mathbf{1 3 a}$ & $\mathrm{MeOH}$ & 50 & $252(13.0)$ & $231(11.3), 375(10.0), 537(2.7)$ \\
$\mathbf{1 3 b}$ & $\mathrm{MeOH}$ & 100 & $250(14.3)$ & $232(12.6), 378(10.3), 549(2.8)$ \\
$\mathbf{2 4}$ & $\mathrm{MeOH}$ & 100 & $264(18.5), 292(17.1)$ & $234(20.6), 352(13.8), 497(2.5)$ \\
$\mathbf{3 5}$ & $\mathrm{MeOH}$ & 100 & $255(28.8), 291^{b}(14.3)$ & $369(9.7), 543(3.0)$ \\
$\mathbf{3 6}$ & $\mathrm{MeOH}$ & & $369(10.1), 540(3.7)$ \\
$\mathbf{3 7}$ & $\mathrm{MeOH}$ & & $391(11.5), 586(5.7)$
\end{tabular}

${ }^{a}$ UV-Vis spectroscopic data are reported for solutions at $25^{\circ} \mathrm{C}$ and reported in $\mathrm{nm}\left(\lambda_{\max }\right)$ and $10^{3} \mathrm{~cm}^{-1} \mathrm{M}^{-1}(\varepsilon)$. The PSS were obtained by irradiation of solutions of the open isomer with light of $312 \mathrm{~nm}$ (Herolab, $6 \mathrm{~W}) .{ }^{b}$ Shoulder. 
Research Systems OptiMelt MPA 100. NMR spectra were recorded on a Bruker Avance $300\left({ }^{1} \mathrm{H} 300.13 \mathrm{MHz},{ }^{13} \mathrm{C} 75.48 \mathrm{MHz}\right)$, Bruker Avance $400\left({ }^{1} \mathrm{H} 400.13 \mathrm{MHz},{ }^{13} \mathrm{C} 100.61 \mathrm{MHz}\right)$ or Avance III $600\left({ }^{1} \mathrm{H} 600.25 \mathrm{MHz},{ }^{13} \mathrm{C} 150.95 \mathrm{MHz}\right)$ instrument. The spectra are referenced against the NMR-solvent, chemical shifts are reported in ppm and coupling constants $J$ are given in $\mathrm{Hz}$. Resonance multiplicity is abbreviated as: s (singlet), d (doublet), $\mathrm{t}$ (triplet), $\mathrm{m}$ (multiplet) and b (broad). Carbon NMR signals are reported with $(+)$ for primary/tertiary, $(-)$ for secondary and $(q)$ for quaternary carbons. The assignment resulted from DEPT, HSQC and HMBC experiments. Mass spectra were recorded on a Finnigan MAT95 (EI-MS), Agilent Q-TOF 6540 UHD (ESI-MS, APCI-MS), Finnigan MAT SSQ 710 A (EI-MS, CI-MS) or ThermoQuest Finnigan TSQ 7000 (ES-MS, APCI-MS) spectrometer. UV/Vis absorption spectroscopy was performed using a Varian Cary BIO 50 UV/Vis/NIR spectrometer. IR-spectra were recorded with a Specac Golden Gate Diamond Single Reflection ATR System in a Bio-Rad FT-IR-Spectrometer Excalibur FTS 3000 and peak positions are reported in wavenumbers $\left(\mathrm{cm}^{-1}\right)$. Standard hand-held lamps were used for visualizing TLC plates and to carry out the ring-closure reactions at $312 \mathrm{~nm}$ (Herolab, $312 \mathrm{~nm}$, $6 \mathrm{~W})$. The ring-opening reactions were performed with the light of a $200 \mathrm{~W}$ tungsten light bulb which was passed through a 420 $\mathrm{nm}$ cut-off filter to eliminate higher energy light or the light of a green LED (CREE-XP green, $530 \mathrm{~nm}, 700 \mathrm{~mA}$ ). The power of the light is given based on the specifications supplied by the company when the lamps were purchased. A light detector was not used to measure the intensity during the irradiation experiments.

\section{3,4-Bis(5-chloro-2-methylthiophen-3-yl)furan-2,5-dione (4)}

To obtain the potassium salt 3, the ester 2 was dissolved in EtOH (2.5 mL mmol ${ }^{-1}$ ) and $\mathrm{KOH}$ (1.0 eq.) was added. After stirring overnight the solvent was removed and the salt 3 was used without further purification. A mixture of the acid 1 (984 $\mathrm{mg}, 5.16 \mathrm{mmol}$ ), the potassium salt $3(1.25 \mathrm{~g}, 5.16 \mathrm{mmol})$ and acetic anhydride (15 $\mathrm{mL}$ ) was heated to $120^{\circ} \mathrm{C}$ for $5 \mathrm{~h}$. The reaction was cooled to room temperature and quenched by adding water $(20 \mathrm{~mL})$. The aqueous phase was extracted with EtOAc $(3 \times 15 \mathrm{~mL})$. The combined organic phases were dried over $\mathrm{MgSO}_{4}$, filtered and the solvent was removed under reduced pressure. The crude product was purified by automated flash column chromatography $\left(\mathrm{PE} / \mathrm{CH}_{2} \mathrm{Cl}_{2}, 30-60 \%\right.$ $\left.\mathrm{CH}_{2} \mathrm{Cl}_{2}\right)$ to yield the maleic anhydride $4(1.07 \mathrm{~g}, 58 \%)$ as gray solid. $R_{\mathrm{f}}: 0.45$ (PE/ $\left.\mathrm{CH}_{2} \mathrm{Cl}_{2}: 1 / 1\right)$; m.p.: $205{ }^{\circ} \mathrm{C}$; IR (neat) $\nu_{\max }: 3108,2922$, 1843, 1766, 1629, 1539, 1460, 1252, 1047, 1177, 990, 919; ${ }^{1} \mathrm{H}-\mathrm{NMR}$ (300 MHz, DMSO- $\left.d_{6}\right): \delta=1.94\left(\mathrm{~s}, 6 \mathrm{H}\right.$, thiophene- $\left.\mathrm{CH}_{3}\right), 7.03(\mathrm{~s}, 2 \mathrm{H}$, thiophene- $H$ ); ${ }^{13} \mathrm{C}-\mathrm{NMR}$ (75 MHz, DMSO- $d_{6}$ ): $\delta=14.1(+), 125.2$ (q), 125.3 (q), $127.2(+), 134.5$ (q), 141.4 (q), 164.5 (q); HRMS (ESI): calcd for $\mathrm{C}_{14} \mathrm{H}_{9} \mathrm{Cl}_{2} \mathrm{O}_{3} \mathrm{~S}_{2}(\mathrm{M}+\mathrm{H})^{+}$358.9365; found 358.9362 .

\section{$N^{2}$-((Benzyloxy)carbonyl)- $N^{5}$-(3,4-bis(5-chloro-2-} methylthiophen-3-yl)-2,5-dioxo-2,5-dihydro-1 $H$-pyrrol-1-yl)-Lglutamine (6)

Maleic anhydride 4 (40 $\mathrm{mg}, 0.11 \mathrm{mmol}$ ) was added to a solution of acid hydrazide 5 (30 mg, $0.10 \mathrm{mmol})$ in THF ( $1 \mathrm{~mL}$ ) in a crimp top vial. After heating to $85{ }^{\circ} \mathrm{C}$ for $16 \mathrm{~h}$ the reaction was quenched with $1 \mathrm{M}$ aqueous $\mathrm{HCl}$ solution $(1 \mathrm{~mL})$ and water (1 $\mathrm{mL})$. The aqueous phase was extracted with EtOAc $(3 \times 5 \mathrm{~mL})$. The combined organic phases were dried over $\mathrm{MgSO}_{4}$ and the solvent was removed under reduced pressure. Purification by automated reversed phase flash column chromatography ( $\mathrm{MeCN} / \mathrm{H}_{2} \mathrm{O}$ with $0.05 \% \mathrm{TFA}, 10-95 \% \mathrm{MeCN}$ ) yielded compound 6 (40 mg, 63\%) as orange solid. $R_{\mathrm{f}}: 0.01$ (EtOAc); m.p.: $103{ }^{\circ} \mathrm{C}$; IR (neat) $\nu_{\max }: 3250,2924,1789,1727,1522,1462,1434,1215,1175$, 990; ${ }^{1} \mathrm{H}-\mathrm{NMR}\left(300 \mathrm{MHz}, \mathrm{DMSO}-d_{6}\right): \delta=1.74-1.88(\mathrm{~m}, 1 \mathrm{H}$, $\left.\mathrm{CO}-\left(\mathrm{CH}_{2}\right)_{2}-\mathrm{CH}\right), 1.94\left(\mathrm{~s}, 6 \mathrm{H}\right.$, thiophene- $\left.\mathrm{CH}_{3}\right), 2.00-2.10(\mathrm{~m}, 1 \mathrm{H}$, $\left.\mathrm{CO}-\left(\mathrm{CH}_{2}\right)_{2}-\mathrm{CH}\right), 2.38-2.48\left(\mathrm{~m}, 2 \mathrm{H}, \mathrm{CO}-\left(\mathrm{CH}_{2}\right)_{2}-\mathrm{CH}\right), 4.03(\mathrm{dt}, J=$ 9.0, $\left.4.8 \mathrm{~Hz}, 1 \mathrm{H}, \mathrm{CH}_{2}-\mathrm{CH}-\mathrm{NH}\right), 5.05$ (s, 2H, O-CH $\left.-\mathrm{Ph}\right), 7.03$ (s, $2 \mathrm{H}$, thiophene- $H$ ), 7.31-7.40 (m, $5 \mathrm{H}, \mathrm{Ph}-H), 7.68(\mathrm{~d}, J=8.0 \mathrm{~Hz}$, 1H, CH-NH-CO), 10.63 (s, 1H, N-NH-CO), 12.70 (bs, $1 \mathrm{H}$, $\mathrm{COO} H) ;{ }^{13} \mathrm{C}-\mathrm{NMR}\left(75 \mathrm{MHz}, \mathrm{DMSO}-d_{6}\right): \delta=14.2(+), 26.1(-), 29.1$ $(-), 53.1(+), 65.4(-), 125.0(\mathrm{q}), 125.8(\mathrm{q}), 127.6(+), 127.6(+)$, $127.7(+), 128.3(+), 131.2(\mathrm{q}), 136.8(\mathrm{q}), 140.6(\mathrm{q}), 156.1$ (q), 166.9 (q), 170.8 (q), 173.4 (q); HRMS (ESI): calcd for $\mathrm{C}_{27} \mathrm{H}_{24} \mathrm{Cl}_{2} \mathrm{~N}_{3} \mathrm{O}_{7} \mathrm{~S}_{2}$ $(\mathrm{M}+\mathrm{H})^{+}$636.0427; found 636.0428.

\section{(S)-2-(((Benzyloxy)carbonyl)amino)-6-(3,4-bis(5-chloro-2- methylthiophen-3-yl)-2,5-dioxo-2,5-dihydro-1 $H$-pyrrol-1-yl) hexanoic acid (7)}

Triethylamine $(131 \mu \mathrm{L}, 0.95 \mathrm{mmol})$ was added to a suspension of maleic anhydride 4 (97 mg, $0.27 \mathrm{mmol}$ ) and Cbz-Lys-OH (83 $\mathrm{mg}, 0.30 \mathrm{mmol})$ in THF ( $5 \mathrm{~mL})$ in a crimp top vial. After heating to $85{ }^{\circ} \mathrm{C}$ for $16 \mathrm{~h}$ the reaction was quenched with $1 \mathrm{M}$ aqueous $\mathrm{HCl}$ solution $(3 \mathrm{~mL})$ and water $(3 \mathrm{~mL})$. The aqueous phase was extracted with EtOAc $(3 \times 15 \mathrm{~mL})$. The combined organic phases were dried over $\mathrm{MgSO}_{4}$ and the solvent was removed under reduced pressure. Purification by automated reversed phase flash column chromatography $\left(\mathrm{MeCN} / \mathrm{H}_{2} \mathrm{O}\right.$ with $0.05 \%$ TFA, 50$100 \% \mathrm{MeCN}$ ) yielded compound 7 (138 $\mathrm{mg}, 82 \%)$ as orange solid. $R_{\mathrm{f}}: 0.09$ (PE/EtOAc: $\left.1 / 1\right)$; m.p.: $88^{\circ} \mathrm{C}$; IR (neat) $\nu_{\max }: 3351$, 3095, 2932, 2870, 1698, 1526, 1438, 1404, 1211, 989; ${ }^{1} \mathrm{H}-\mathrm{NMR}$ (400 MHz, DMSO- $\left.d_{6}\right): \delta=1.29-1.43\left(\mathrm{~m}, 2 \mathrm{H}, \mathrm{CH}_{2}-\left(\mathrm{CH}_{2}\right)_{3}-\mathrm{CH}\right)$, 1.51-1.65 (m, 3H, $\left.\mathrm{CH}_{2}-\left(\mathrm{CH}_{2}\right)_{3}-\mathrm{CH}\right), 1.67-1.79(\mathrm{~m}, 1 \mathrm{H}$, $\left.\mathrm{CH}_{2}-\left(\mathrm{CH}_{2}\right)_{3}-\mathrm{CH}\right), 1.91$ (s, 6H, thiophene- $\left.\mathrm{CH}_{3}\right), 3.49(\mathrm{t}, J=7.1$ $\left.\mathrm{Hz}, 2 \mathrm{H}, \mathrm{N}-\mathrm{CH}_{2}-\left(\mathrm{CH}_{2}\right)_{3}\right), 3.93(\mathrm{dt}, J=7.9,4.6 \mathrm{~Hz}, 1 \mathrm{H}, \mathrm{NH}-\mathrm{CH}-$ $\mathrm{CH}_{2}$ ), 5.02 (s, 2H, O- $\left.\mathrm{CH}_{2}-\mathrm{Ph}\right), 7.00$ (s, 2H, thiophene- $H$ ), 7.287.37 (m, 5H, Ph- $H$ ), 7.57 (d, $J=8.0 \mathrm{~Hz}, 1 \mathrm{H}, \mathrm{CO}-\mathrm{N} H-\mathrm{CH}), 12.55$ $(\mathrm{s}, 1 \mathrm{H}, \mathrm{COOH}) ;{ }^{13} \mathrm{C}-\mathrm{NMR}\left(101 \mathrm{MHz}, \mathrm{DMSO}-d_{6}\right): \delta=14.1(+), 22.8$ $(-), 27.4(-), 30.2(-), 37.7(-), 53.5(+), 65.3(-), 124.6(\mathrm{q}), 126.4$ (q), $127.6(+), 127.7(+), 127.7(+), 128.2(+), 132.3(q), 136.9$ (q), 139.8 (q), 169.6 (q), 173.8 (q); HRMS (ESI): calcd for $\mathrm{C}_{28} \mathrm{H}_{27} \mathrm{Cl}_{2^{-}}$ $\mathrm{N}_{2} \mathrm{O}_{6} \mathrm{~S}_{2}(\mathrm{M}+\mathrm{H})^{+}$621.0682; found 621.0684.

\section{$N$-(3,4-Bis(5-chloro-2-methylthiophen-3-yl)-2,5-dioxo-2,5- dihydro-1H-pyrrol-1-yl)acetamide (8)}

Hydrazine hydrate $(39 \mu \mathrm{L}, 0.81 \mathrm{mmol})$ was added to a solution of maleic anhydride 4 (97 $\mathrm{mg}, 0.27 \mathrm{mmol})$ in acetic acid $(3.5 \mathrm{~mL})$. The reaction mixture was heated to $100{ }^{\circ} \mathrm{C}$ for $20 \mathrm{~h}$ and then water $(10 \mathrm{~mL})$ was added. The aqueous phase was extracted with EtOAc $(3 \times 10 \mathrm{~mL})$. The combined organic phases were dried over $\mathrm{MgSO}_{4}$ and the solvent was evaporated in vacuo. Purification by automated flash column chromatography (PE/EtOAc, 
15-50\% EtOAc) yielded compound 8 (81 $\mathrm{mg}, 72 \%)$ as orange solid. $R_{\mathrm{f}}: 0.23$ (PE/EtOAc: $2 / 1$ ); m.p.: $131{ }^{\circ} \mathrm{C}$; IR (neat) $\nu_{\max }: 3328$, 3088, 2924, 2359, 1717, 1702, 1510, 1429, 1255, 1193, 988; ${ }^{1} \mathrm{H}-$ NMR (400 MHz, DMSO- $d_{6}$ ): $\delta=1.95\left(\mathrm{~s}, 6 \mathrm{H}\right.$, thiophene- $\mathrm{CH}_{3}$ ), $2.03\left(\mathrm{~s}, 3 \mathrm{H}, \mathrm{CO}-\mathrm{CH}_{3}\right), 7.01$ (s, 2H, thiophene- $\left.H\right), 10.58(\mathrm{~s}, 1 \mathrm{H}$, $\mathrm{N} H) ;{ }^{13} \mathrm{C}-\mathrm{NMR}\left(101 \mathrm{MHz}, \mathrm{DMSO}-d_{6}\right): \delta=14.2(+), 20.0(+), 125.0$ (q), 125.8 (q), 127.6 (+), 131.3 (q), 140.6 (q), 166.9 (q), 168.5 (q); HRMS (ESI): calcd for $\mathrm{C}_{16} \mathrm{H}_{13} \mathrm{Cl}_{2} \mathrm{~N}_{2} \mathrm{O}_{3} \mathrm{~S}_{2}(\mathrm{M}+\mathrm{H})^{+}$414.9739; found 414.9741 .

\section{3,4-Bis(5-chloro-2-methylthiophen-3-yl)-1-methyl-1H-pyrrole- 2,5-dione (9)}

Maleic anhydride 4 (54 mg, $0.15 \mathrm{mmol}$ ) and 1,2-dimethylhydrazine dihydrochloride ( $60 \mathrm{mg}, 0.45 \mathrm{mmol}$ ) were heated to 160 ${ }^{\circ} \mathrm{C}$ for $12 \mathrm{~h}$ in PEG400 ( $2 \mathrm{~mL}$ ) in a crimp top vial. Then water (15 $\mathrm{mL}$ ) was added and the aqueous phase was extracted with EtOAc $(3 \times 15 \mathrm{~mL})$. The combined organic phases were washed with brine $(50 \mathrm{~mL})$, dried over $\mathrm{MgSO}_{4}$ and the solvent was removed under reduced pressure. Purification by automated flash column chromatography (PE/EtOAc, 3-10\% EtOAc) yielded compound 9 (48 mg, 86\%) as orange foam. $R_{\mathrm{f}}: 0.32$ (PE/EtOAc: 19/1); IR (neat) $\nu_{\text {max }}: 3098,2924,2851,1765,1697,1435,1386$, 1250, 1174, 980; ${ }^{1} \mathrm{H}-\mathrm{NMR}\left(300 \mathrm{MHz}, \mathrm{CDCl}_{3}\right): \delta=1.93(\mathrm{~s}, 6 \mathrm{H}$, thiophene- $\left.\mathrm{CH}_{3}\right), 3.13\left(\mathrm{~s}, 3 \mathrm{H}, \mathrm{N}-\mathrm{CH}_{3}\right), 6.89(\mathrm{~s}, 2 \mathrm{H}$, thiophene- $H$ ); ${ }^{13} \mathrm{C}-\mathrm{NMR}\left(75 \mathrm{MHz}, \mathrm{CDCl}_{3}\right.$ ): $\delta=14.9(+), 24.4(+), 126.0$ (q), 127.2 $(+), 127.2$ (q), 132.7 (q), 140.2 (q), 170.2 (q); HRMS (ESI): calcd for $\mathrm{C}_{15} \mathrm{H}_{12} \mathrm{Cl}_{2} \mathrm{NO}_{2} \mathrm{~S}_{2}(\mathrm{M}+\mathrm{H})^{+}$373.9649; found 373.9652 .

\section{Allyl ((4-(2-amino-2-oxoacetyl)-5-methylthiophen-2-yl)methyl) carbamate (10)}

To a solution of oxoacetate $18(282 \mathrm{mg}, 0.95 \mathrm{mmol})$ in THF (5 $\mathrm{mL})$ was added a $\mathrm{NH}_{4} \mathrm{OH}$ solution $\left(32 \%\right.$ in $\left.\mathrm{H}_{2} \mathrm{O}\right)(1.18 \mathrm{~mL}, 9.50$ $\mathrm{mmol}$ ) at $0{ }^{\circ} \mathrm{C}$. The reaction was stirred for $90 \mathrm{~min}$ at room temperature and then quenched with water $(5 \mathrm{~mL})$. The aqueous phase was extracted with EtOAc $(3 \times 10 \mathrm{~mL})$. The combined organic phases were dried over $\mathrm{MgSO}_{4}$ and the solvent was removed under reduced pressure. Compound $\mathbf{1 0}$ (253 $\mathrm{mg}, 94 \%$ ) was obtained as yellow solid and used without further purification. $R_{\mathrm{f}}$ : 0.21 (PE/EtOAc: 1/1); m.p.: $108{ }^{\circ} \mathrm{C}$; IR (neat) $\nu_{\max }: 3402,3301,3167,2962,1750,1686,1649,1535,1460$, 1254, 1047, 796; ${ }^{1} \mathrm{H}-\mathrm{NMR}\left(400 \mathrm{MHz}, \mathrm{CDCl}_{3}\right): \delta=2.70(\mathrm{~s}, 3 \mathrm{H}$, thiophene- $\left.\mathrm{CH}_{3}\right), 4.44\left(\mathrm{~d}, \mathrm{~J}=6.1 \mathrm{~Hz}, 2 \mathrm{H}\right.$, thiophene- $\left.\mathrm{CH}_{2} \mathrm{NH}\right)$, 4.59 (d, $\left.J=5.1 \mathrm{~Hz}, 2 \mathrm{H}, \mathrm{CH}_{2}=\mathrm{CHCH}_{2} \mathrm{O}\right), 5.21$ (dd, $J=10.4,0.5$ $\left.\mathrm{Hz}, 1 \mathrm{H}, \mathrm{CH}_{2}=\mathrm{CHCH}_{2}\right), 5.24-5.43\left(\mathrm{~m}, 2 \mathrm{H}, \mathrm{CH}_{2}=\mathrm{CHCH}_{2}\right.$ and $\mathrm{NH}$ ), 5.90 (ddt, $J=16.2,10.7,5.5 \mathrm{~Hz}, 1 \mathrm{H}, \mathrm{CH}_{2}=\mathrm{CHCH}_{2}$ ), 6.05 (bs, $\left.1 \mathrm{H}, \mathrm{NH}_{2}\right), 7.06$ (bs, $\left.1 \mathrm{H}, \mathrm{NH}_{2}\right), 7.86(\mathrm{~s}, 1 \mathrm{H}$, thiophene- $H) ;{ }^{13} \mathrm{C}-$ NMR (101 MHz, $\left.\mathrm{CDCl}_{3}\right): \delta=16.7(+), 39.8(-), 65.9(-), 117.9$ $(-), 129.0(+), 130.8$ (q), $132.7(+), 137.0$ (q), 155.7 (q), 156.1 (q), 164.4 (q), 182.1 (q); HRMS (ESI): calcd for $\mathrm{C}_{12} \mathrm{H}_{18} \mathrm{~N}_{3} \mathrm{O}_{4} \mathrm{~S}$ (M + $\left.\mathrm{NH}_{4}\right)^{+}$300.1013; found 300.1012.

\section{Methyl 4-(2-methoxy-2-oxoethyl)-5-methylthiophene-2- carboxylate (11)}

Thallium trinitrate (850 mg, $1.91 \mathrm{mmol})$ and $70 \% \mathrm{HClO}_{4}(0.30$ $\mathrm{mL}$ ) were added to a suspension of 21 (316 $\mathrm{mg}, 1.59 \mathrm{mmol})$ in $\mathrm{MeOH}(10 \mathrm{~mL})$ at room temperature. After stirring for $24 \mathrm{~h}$ the mixture was concentrated under vacuum and diluted with water $(10 \mathrm{~mL})$. The aqueous phase was extracted with EtOAc $(3 \times 10$ $\mathrm{mL}$ ) and the combined organic layers were dried over $\mathrm{MgSO}_{4}$. The solvent was evaporated and purification of the crude product by automated flash column chromatography (PE/ EtOAc, 3-15\% EtOAc) yielded compound 11 (331 mg, 91\%) as colorless oil. $R_{\mathrm{f}}: 0.34$ (PE/EtOAc: 5/1); IR (neat) $\nu_{\max }: 2997,2953$, 2845, 1736, 1704, 1535, 1457, 1392, 1331, 1291, 1250, 1194, 1132, 1063, 1006, 927, 874, 785, 751; ${ }^{1} \mathrm{H}-\mathrm{NMR}\left(400 \mathrm{MHz}, \mathrm{CDCl}_{3}\right.$ ): $\delta=2.42\left(\mathrm{~s}, 3 \mathrm{H}\right.$, thiophene- $\left.\mathrm{CH}_{3}\right), 3.54\left(\mathrm{~s}, 2 \mathrm{H}\right.$, thiophene- $\mathrm{CH}_{2} \mathrm{C}(\mathrm{O})$ $\left.\mathrm{OCH}_{3}\right), 3.69$ (s, 3H, thiophene- $\left.\mathrm{CH}_{2} \mathrm{C}(\mathrm{O}) \mathrm{OCH}_{3}\right), 3.84(\mathrm{~s}, 3 \mathrm{H}, \mathrm{C}(\mathrm{O})$ $\left.\mathrm{OCH}_{3}\right), 7.60(\mathrm{~s}, 1 \mathrm{H}$, thiophene- $H) ;{ }^{13} \mathrm{C}-\mathrm{NMR}\left(101 \mathrm{MHz}, \mathrm{CDCl}_{3}\right): \delta$ $=13.9(+), 33.9(-), 52.1(+), 52.3(+), 129.2(\mathrm{q}), 130.8(\mathrm{q}), 135.7$ $(+), 144.1$ (q), 162.7 (q), 171.0 (q); HRMS (APCI): calcd for $\mathrm{C}_{10} \mathrm{H}_{12} \mathrm{O}_{4} \mathrm{~S}(\mathrm{M}+\mathrm{H})^{+}$229.0529; found 229.0531.

Methyl 4-(4-(5-((((allyloxy)carbonyl)amino)methyl)-2-methylthiophen-3-yl)-2,5-dioxo-2,5-dihydro-1H-pyrrol-3-yl)-5methylthiophene-2-carboxylate (12)

$\mathrm{KO} t \mathrm{Bu}(1 \mathrm{M}$ in THF, $0.88 \mathrm{~mL}, 0.88 \mathrm{mmol}$ ) was added to a solution of glyoxylamide $10(206 \mathrm{mg}, 0.73 \mathrm{mmol})$ in anhydrous THF ( $5 \mathrm{~mL})$ at $0{ }^{\circ} \mathrm{C}$ under nitrogen atmosphere. After stirring for $90 \mathrm{~min}$ at $0{ }^{\circ} \mathrm{C}$, diester $11(200 \mathrm{mg}, 0.88 \mathrm{mmol})$ in THF $(2 \mathrm{~mL})$ was added at $0{ }^{\circ} \mathrm{C}$ and stirred for 3 days at room temperature. Then the reaction was quenched with $1 \mathrm{M}$ aqueous $\mathrm{HCl}$ solution $(3 \mathrm{~mL})$ and diluted with EtOAc $(10 \mathrm{~mL})$. The organic phase was washed with water $(2 \times 10 \mathrm{~mL})$, brine $(10 \mathrm{~mL})$ and dried over $\mathrm{MgSO}_{4}$. The solvent was removed under reduced pressure and purification of the crude product by automated flash column chromatography (PE/EtOAc, 25-50\% EtOAc) yielded 12 (148 mg, $44 \%$ ) as yellow foam. $R_{\mathrm{f}}$ : 0.20 (PE/EtOAc: $2 / 1$ ); IR (neat) $\nu_{\max }$ : 3289, 3070, 2952, 1703, 1540, 1458, 1339, 1248, 994, 909, 727; ${ }^{1} \mathrm{H}-\mathrm{NMR}\left(400 \mathrm{MHz}, \mathrm{CDCl}_{3}\right): \delta=1.90\left(\mathrm{~s}, 3 \mathrm{H}\right.$, thiophene- $\left.\mathrm{CH}_{3}\right)$; $1.98\left(\mathrm{~s}, 3 \mathrm{H}\right.$, thiophene- $\left.\mathrm{CH}_{3}\right), 3.87\left(\mathrm{~s}, 1 \mathrm{H}, \mathrm{OCH}_{3}\right), 4.45(\mathrm{~d}, J=$ $6.0 \mathrm{~Hz}, 2 \mathrm{H}$, thiophene- $\left.\mathrm{CH}_{2} \mathrm{NH}\right), 4.60(\mathrm{~d}, J=4.9 \mathrm{~Hz}, 2 \mathrm{H}$, $\left.\mathrm{CH}_{2}=\mathrm{CHCH}_{2} \mathrm{O}\right), 5.13-5.27\left(\mathrm{~m}, 2 \mathrm{H}, \mathrm{CH}_{2}=\mathrm{CHCH}_{2}\right.$ and $\mathrm{CH}_{2}-$ $\mathrm{NHCO}$ ), 5.31 (dd, $J=17.2,1.2 \mathrm{~Hz}, 1 \mathrm{H}, \mathrm{CH}_{2}=\mathrm{CHCH}_{2}$ ), 5.92 (ddt, $\left.J=16.2,10.8,5.5 \mathrm{~Hz}, 1 \mathrm{H}, \mathrm{CH}_{2}=\mathrm{CHCH}_{2}\right), 6.90(\mathrm{~s}, 1 \mathrm{H}$, thiophene$H$ ), 7.74 (s, $1 \mathrm{H}$, thiophene- $H$ ), 8.03 (bs, $1 \mathrm{H}, \mathrm{CONHCO}) ;{ }^{13} \mathrm{C}-\mathrm{NMR}$ $\left(101 \mathrm{MHz}, \mathrm{CDCl}_{3}\right): \delta=15.0(+), 15.3(+), 39.9(-), 52.3(+), 65.9$ $(-), 117.9(-), 125.8$ (q), $126.7(+), 127.5$ (q), 130.9 (q), $132.7(+)$, 134.8 (q), $134.9(+), 139.4$ (q), 142.1 (q), 148.6 (q), 156.0 (q), 162.1 (q), 170.0 (q), 170.2 (q); HRMS (ESI): calcd for $\mathrm{C}_{21} \mathrm{H}_{21} \mathrm{~N}_{2} \mathrm{O}_{6} \mathrm{~S}_{2}$ (M $+\mathrm{H})^{+}$461.0838; found 461.0836.

4-(4-(5-(Aminomethyl)-2-methylthiophen-3-yl)-2,5-dioxo-2,5dihydro-1H-pyrrol-3-yl)-5-methylthiophene-2-carboxylic acid (13a) and 4-(4-(5-((((allyloxy)carbonyl)amino)methyl)-2methylthiophen-3-yl)-2,5-dioxo-2,5-dihydro-1H-pyrrol-3-yl)-5methylthiophene-2-carboxylic acid (13b)

A solution of $\mathrm{BBr}_{3}\left(1 \mathrm{M}\right.$ in $\left.\mathrm{CH}_{2} \mathrm{Cl}_{2}, 2.0 \mathrm{~mL}, 2.00 \mathrm{mmol}\right)$ was added to a solution of compound 12 (92 $\mathrm{mg}, 0.20 \mathrm{mmol}$ ) in anhydrous $\mathrm{CH}_{2} \mathrm{Cl}_{2}(6 \mathrm{~mL})$ in a crimp top vial. The mixture was heated to $40^{\circ} \mathrm{C}$ for $5 \mathrm{~h}$. Then water $(4 \mathrm{~mL})$ was added via syringe and the suspension was stirred at $40{ }^{\circ} \mathrm{C}$ for additional $30 \mathrm{~min}$. After cooling to room temperature the solvent was removed at 
the rotary evaporator. Purification by automated reversed phase flash column chromatography $\left(\mathrm{MeCN} / \mathrm{H}_{2} \mathrm{O}\right.$ with $0.05 \%$ TFA, 3$100 \% \mathrm{MeCN}$ ) yielded compound 13a (34 mg, 47\%) as yellow solid and compound 13b (18 mg, 20\%) as yellow solid.

\section{Analytical data of 13a}

$R_{\mathrm{f}}: 0.02$ (PE/EtOAc: 1/1); m.p.: $173{ }^{\circ} \mathrm{C}$; IR (neat) $\nu_{\text {max }}: 3008,2924$, 1766, 1712, 1681, 1545, 1463, 1344, 1188, 1137, 1001, 839, 799, 756, 723; ${ }^{1} \mathrm{H}-\mathrm{NMR}(600 \mathrm{MHz}, \mathrm{MeOD}): \delta=2.00$ (s, 3H, thiophene$\left.\mathrm{CH}_{3}\right), 2.08\left(\mathrm{~s}, 3 \mathrm{H}\right.$, thiophene- $\left.\mathrm{CH}_{3}\right), 4.27(\mathrm{~s}, 2 \mathrm{H}$, thiophene$\left.\mathrm{CH}_{2} \mathrm{NH}\right), 7.18$ (s, $1 \mathrm{H}$, thiophene- $H$ ), 7.64 (s, $1 \mathrm{H}$, thiophene- $H$ ); ${ }^{13} \mathrm{C}-\mathrm{NMR}$ (151 MHz, MeOD): $\delta=14.7(+), 15.3(+), 38.5(-), 128.5$ (q), $129.3(\mathrm{q}), 132.2(+), 132.8(\mathrm{q}), 133.1$ (q), $135.0(\mathrm{q}), 135.7$ (q), $136.3(+), 144.6$ (q), 149.7 (q), 164.6 (q), 172.4 (q), 172.6 (q); HRMS (ESI): calcd for $\mathrm{C}_{16} \mathrm{H}_{15} \mathrm{~N}_{2} \mathrm{O}_{4} \mathrm{~S}_{2}(\mathrm{M}+\mathrm{H})^{+} 363.0469$; found 363.0468 .

\section{Analytical data of 13b}

$R_{\mathrm{f}}: 0.04$ (PE/EtOAc: 1/1); m.p.: $94{ }^{\circ} \mathrm{C}$; IR (neat) $\nu_{\max }: 2926,1981$, 1769, 1709, 1544, 1459, 1344, 1246, 1185, 1150, 1049, 991, 849, 762; ${ }^{1} \mathrm{H}-\mathrm{NMR}\left(300 \mathrm{MHz}, \mathrm{CD}_{3} \mathrm{CN}\right): \delta=1.93$ (s, 3H, thiophene$\left.\mathrm{CH}_{3}\right), 1.97$ (s, 3H, thiophene- $\left.\mathrm{CH}_{3}\right), 4.33(\mathrm{~d}, J=6.3 \mathrm{~Hz}, 2 \mathrm{H}$, thiophene- $\left.\mathrm{CH}_{2} \mathrm{NH}\right), 4.52\left(\mathrm{~d}, J=5.3 \mathrm{~Hz}, 2 \mathrm{H}, \mathrm{CH}_{2}=\mathrm{CHCH}_{2} \mathrm{O}\right)$, $5.18\left(\mathrm{dd}, J=10.5,1.4 \mathrm{~Hz}, 1 \mathrm{H}, \mathrm{CH}_{2}=\mathrm{CHCH}_{2}\right), 5.27(\mathrm{dd}, J=17.3$, $\left.1.6 \mathrm{~Hz}, 1 \mathrm{H}, \mathrm{CH}_{2}=\mathrm{CHCH}_{2}\right)$, 5.74-6.05 (m, $\left.1 \mathrm{H}, \mathrm{CH}_{2}=\mathrm{CHCH}_{2}\right)$, 6.14 (bs, $\left.1 \mathrm{H}, \mathrm{CH}_{2} \mathrm{NHCO}\right), 6.79(\mathrm{~s}, 1 \mathrm{H}$, thiophene- $H), 7.60(\mathrm{~s}, 1 \mathrm{H}$, thiophene- $H$ ), 8.80 (bs, $1 \mathrm{H}, \mathrm{COOH}) ;{ }^{13} \mathrm{C}-\mathrm{NMR}\left(75 \mathrm{MHz}, \mathrm{CD}_{3} \mathrm{CN}\right)$ : $\delta=14.8(+), 15.2(+), 40.1(-), 66.0(-), 117.5(-), 127.2(\mathrm{q}), 127.4$ $(+), 129.1(\mathrm{q}), 131.4(\mathrm{q}), 134.1(\mathrm{q}), 134.4(+), 136.2(+), 136.2(\mathrm{q})$, 141.2 (q), 141.8 (q), 149.6 (q), 157.1 (q), 162.9 (q), 171.6 (q), 171.7 (q); HRMS (ESI): calcd for $\mathrm{C}_{20} \mathrm{H}_{18} \mathrm{~N}_{2} \mathrm{O}_{6} \mathrm{~S}_{2}(\mathrm{M}+\mathrm{H})^{+}$447.0679; found 447.0676 .

\section{Alternative procedure to obtain 13b}

Compound 12 (40 mg, $0.09 \mathrm{mmol}$ ) was dissolved in acetone (10 $\mathrm{mL}$ ) and LiI (350 mg, $2.60 \mathrm{mmol}$ ) was added. The mixture was heated to $100{ }^{\circ} \mathrm{C}$ overnight. After cooling to room temperature it was quenched with $1 \mathrm{M}$ aqueous $\mathrm{HCl}$ solution $(5 \mathrm{~mL})$ and diluted with $\mathrm{CH}_{2} \mathrm{Cl}_{2}(5 \mathrm{~mL})$. The phases were separated and the aqueous phase was extracted with $\mathrm{CH}_{2} \mathrm{Cl}_{2}(3 \times 5 \mathrm{~mL})$. The combined organic phases were dried over $\mathrm{Na}_{2} \mathrm{SO}_{4}$ and the solvent was removed at the rotary evaporator. Automated reversed phase flash column chromatography $\left(\mathrm{MeCN} / \mathrm{H}_{2} \mathrm{O}\right.$ with $0.05 \%$ TFA, 3-100\% MeCN) yielded compound 13b (14 mg, $35 \%$ ) as yellow solid.

\section{Allyl ((5-methylthiophen-2-yl)methyl)carbamate (17)}

LAH $(2.78 \mathrm{~g}, 73.2 \mathrm{mmol})$ was added in portions to a solution of nitrile $16(3.01 \mathrm{~g}, 24.4 \mathrm{mmol})$ in anhydrous $\mathrm{Et}_{2} \mathrm{O}(250 \mathrm{~mL})$ at $0{ }^{\circ} \mathrm{C}$ under nitrogen atmosphere. After stirring for $4 \mathrm{~h}$ at room temperature the reaction was quenched with water $(80 \mathrm{~mL})$ and saturated aqueous $\mathrm{NaHCO}_{3}$ solution $(50 \mathrm{~mL})$ at $0{ }^{\circ} \mathrm{C}$. The suspension was filtered and the aqueous phase was extracted with $\mathrm{Et}_{2} \mathrm{O}(3 \times 80 \mathrm{~mL})$. The combined organic phases were dried over $\mathrm{MgSO}_{4}$ and concentrated in vacuo. Then the residue was dissolved in anhydrous THF $(100 \mathrm{~mL})$ and pyridine $(2.47 \mathrm{~mL}$, $30.50 \mathrm{mmol}$ ) was added at $0{ }^{\circ} \mathrm{C}$. Within $1 \mathrm{~h}$ allyl chloroformate (4.02 $\mathrm{mL}, 37.82 \mathrm{mmol}$ ) in anhydrous THF ( $5 \mathrm{~mL}$ ) was dropped to the solution via a syringe pump at $0{ }^{\circ} \mathrm{C}$. After stirring for $14 \mathrm{~h}$ at room temperature the reaction was quenched cautiously with water $(50 \mathrm{~mL})$ and extracted with EtOAc $(3 \times 30 \mathrm{~mL})$. The combined organic phases were dried over $\mathrm{MgSO}_{4}$ and the solvent was removed under reduced pressure. Purification of the crude product by automated flash column chromatography (PE/EtOAc, 8-15\% EtOAc) yielded 17 (3.40 g, 66\%) as yellow oil. $R_{\mathrm{f}}: 0.20$ (PE/EtOAc: 9/1); IR (neat) $\nu_{\max }: 3335,3073,2922,1695$, 1514, 1426, 1236, 982, 799; ${ }^{1} \mathrm{H}-\mathrm{NMR}$ (400 MHz, $\left.\mathrm{CDCl}_{3}\right): \delta=2.44$ $\left(\mathrm{s}, 3 \mathrm{H}\right.$, thiophene- $\left.\mathrm{CH}_{3}\right), 4.44(\mathrm{~d}, \mathrm{~J}=5.7 \mathrm{~Hz}, 2 \mathrm{H}$, thiophene$\mathrm{CH}_{2} \mathrm{NH}$ ), 4.61 (d, $J=5.3 \mathrm{~Hz}, 2 \mathrm{H}, \mathrm{CH}_{2}=\mathrm{CHCH}_{2} \mathrm{O}$ ), 5.05 (bs, $1 \mathrm{H}$, $\mathrm{NH}), 5.25\left(\mathrm{dd}, J=10.4,0.3 \mathrm{~Hz}, 1 \mathrm{H}, \mathrm{CH}_{2}=\mathrm{CHCH}_{2}\right.$ ), 5.30 (dd, $J=$ 17.1, $\left.1.2 \mathrm{~Hz}, 1 \mathrm{H}, \mathrm{CH}_{2}=\mathrm{CHCH}_{2}\right), 5.92(\mathrm{ddt}, J=16.4,10.8,5.7 \mathrm{~Hz}$, $1 \mathrm{H}, \mathrm{CH}_{2}=\mathrm{CHCH}_{2}$ ), 6.57 (dd, $J=3.1,1.0 \mathrm{~Hz}, 1 \mathrm{H}, 4$-thiophene- $H$ ), $6.74(\mathrm{~d}, J=3.1 \mathrm{~Hz}, 1 \mathrm{H}, 3$-thiophene- $H) ;{ }^{13} \mathrm{C}-\mathrm{NMR}(101 \mathrm{MHz}$, $\left.\mathrm{CDCl}_{3}\right): \delta=15.4(+), 40.1(-), 65.7(-), 117.8(-), 124.8(+), 125.7$ $(+), 132.8(+), 138.8$ (q), 139.9 (q), 155.9 (q); HRMS (ESI): calcd for $\mathrm{C}_{10} \mathrm{H}_{14} \mathrm{NO}_{2} \mathrm{~S}(\mathrm{M}+\mathrm{H})^{+}$212.0740; found 212.0740.

\section{Methyl 2-(5-((((allyloxy)carbonyl)amino)methyl)-2- methylthiophen-3-yl)-2-oxoacetate (18)}

Carbamate 17 (169 mg, $0.80 \mathrm{mmol}$ ) and methyl chlorooxoacetate $(81 \mu \mathrm{L}, 0.88 \mathrm{mmol})$ were dissolved in anhydrous $\mathrm{CH}_{2} \mathrm{Cl}_{2}(6 \mathrm{~mL})$ under nitrogen atmosphere. Then aluminum chloride (427 mg, $3.20 \mathrm{mmol}$ ) was added in portions at $0{ }^{\circ} \mathrm{C}$ and the suspension was stirred for $20 \mathrm{~h}$ at room temperature. The reaction was quenched with saturated aqueous $\mathrm{NaHCO}_{3}$ solution $(1 \mathrm{~mL})$ at $0{ }^{\circ} \mathrm{C}$ and diluted with water $(5 \mathrm{~mL})$. The aqueous phase was extracted with $\mathrm{CH}_{2} \mathrm{Cl}_{2}(3 \times 5 \mathrm{~mL})$, the combined organic layers were washed with brine $(10 \mathrm{~mL})$ and dried over $\mathrm{MgSO}_{4}$. After evaporation of the solvent the crude product was purified by automated flash column chromatography (PE/ EtOAc, 15-40\% EtOAc) to obtain 18 (117 $\mathrm{mg}, 49 \%)$ as brown oil. $R_{\mathrm{f}}: 0.22$ (PE/EtOAc: 3/1); IR (neat) $\nu_{\max }: 3395,2954,1726$, 1670, 1517, 1434, 1242, 1200, 1112, 984, 757; ${ }^{1} \mathrm{H}-\mathrm{NMR}(400 \mathrm{MHz}$, $\left.\mathrm{CDCl}_{3}\right): \delta=2.70\left(\mathrm{~s}, 3 \mathrm{H}\right.$, thiophene- $\left.\mathrm{CH}_{3}\right), 3.91\left(\mathrm{~s}, 3 \mathrm{H}, \mathrm{OCH}_{3}\right), 4.42$ $\left(\mathrm{d}, J=6.1 \mathrm{~Hz}, 2 \mathrm{H}\right.$, thiophene- $\left.\mathrm{CH}_{2} \mathrm{NH}\right), 4.58(\mathrm{~d}, J=5.3 \mathrm{~Hz}, 2 \mathrm{H}$, $\left.\mathrm{CH}_{2}=\mathrm{CHCH}_{2} \mathrm{O}\right), 5.16-5.34\left(\mathrm{~m}, 3 \mathrm{H}, \mathrm{CH}_{2}=\mathrm{CHCH}_{2}\right.$ and $\left.\mathrm{NH}\right), 5.90$ (ddt, $\left.J=16.3,10.8,5.6 \mathrm{~Hz}, 1 \mathrm{H}, \mathrm{CH}_{2}=\mathrm{CHCH}_{2}\right), 7.32(\mathrm{~s}, 1 \mathrm{H}$, thiophene- $H$ ); ${ }^{13} \mathrm{C}-\mathrm{NMR}\left(101 \mathrm{MHz}, \mathrm{CDCl}_{3}\right): \delta=16.3(+), 39.7(-)$, $52.8(+), 65.9(-), 118.0(-), 127.5(+), 131.0(\mathrm{q}), 132.6(+), 138.0$ (q), 154.8 (q), 156.1 (q), 164.0 (q), 180.0 (q); HRMS (ESI): calcd for $\mathrm{C}_{13} \mathrm{H}_{16} \mathrm{NO}_{5} \mathrm{~S}(\mathrm{M}+\mathrm{H})^{+}$298.0744; found 298.0744.

\section{Methyl 4-acetyl-5-methylthiophene-2-carboxylate (21)}

Thiophene 20 (800 mg, $5.12 \mathrm{mmol})$ and acetyl chloride $(550 \mu \mathrm{L}$, $7.68 \mathrm{mmol})$ were dissolved in purified anhydrous $\mathrm{CHCl}_{3}(10 \mathrm{~mL})$ under nitrogen atmosphere. After cooling to $0{ }^{\circ} \mathrm{C}$ aluminum chloride $(2.05 \mathrm{~g}, 15.4 \mathrm{mmol})$ was added in small portions. The yellow suspension was heated to $45{ }^{\circ} \mathrm{C}$ overnight upon turning bright red, then the reaction was quenched with ice/water and the aqueous phase was extracted with EtOAc $(3 \times 10 \mathrm{~mL})$. The combined organic phases were washed with a saturated 
aqueous solution of $\mathrm{NaHCO}_{3}(10 \mathrm{~mL})$ and brine $(10 \mathrm{~mL})$. The organic phase was dried over $\mathrm{MgSO}_{4}$ and the solvent was evaporated. The crude product was purified by automated flash column chromatography (PE/EtOAc, 5-25\% EtOAc) and compound 21 (781 mg, 77\%) was obtained as colorless solid. $R_{\mathrm{f}}$ : 0.41 (PE/EtOAc: 3/1); m.p.: $84{ }^{\circ} \mathrm{C}$; IR (neat) $\nu_{\text {max }}$ : 3007, 2957, 1717, 1678, 1539, 1457, 1439, 1254, 1233, 1074, 1021, 745; ${ }^{1} \mathrm{H}-$ NMR (300 MHz, $\left.\mathrm{CDCl}_{3}\right): \delta=2.52\left(\mathrm{~s}, 3 \mathrm{H}\right.$, thiophene- $\left.\mathrm{CH}_{3}\right), 2.76$ $\left(\mathrm{s}, 3 \mathrm{H}\right.$, acetyl- $\left.\mathrm{CH}_{3}\right), 3.88\left(\mathrm{~s}, 3 \mathrm{H}, \mathrm{OCH}_{3}\right), 8.03(\mathrm{~s}, 1 \mathrm{H}$, thiophene- $H)$; ${ }^{13} \mathrm{C}-\mathrm{NMR}\left(75 \mathrm{MHz}, \mathrm{CDCl}_{3}\right): \delta=16.8(+), 29.6(+), 52.3(+), 128.5$ (q), 135.0 (+), 136.3 (q), 155.8 (q), 162.0 (q), 193.7 (q); HRMS (APCI): calcd for $\mathrm{C}_{9} \mathrm{H}_{10} \mathrm{O}_{3} \mathrm{~S}(\mathrm{M}+\mathrm{H})^{+}$199.0423; found 199.0424.

\section{General procedure A: Suzuki coupling}

To a suspension of $\mathrm{Pd}_{2}(\mathrm{dba})_{3}(5 \mathrm{~mol} \%)$, XPhos (10 mol\%), the appropriate boronic acid (1.5 eq.) and $\mathrm{K}_{3} \mathrm{PO}_{4}$ (1.5 eq.) in 1,4dioxane $(0.5 \mathrm{M})$ the appropriate ester (1.0 eq.) was added. The resulting mixture was heated to $100{ }^{\circ} \mathrm{C}$ and stirred overnight. After cooling to room temperature the reaction mixture was diluted with EtOAc and the organic phase was washed two times with water. The organic phase was dried over $\mathrm{MgSO}_{4}$, filtered and the solvent was removed under reduced pressure.

\section{General procedure B: aminolysis}

An $\mathrm{NH}_{4} \mathrm{OH}$ solution (25\% in $\mathrm{H}_{2} \mathrm{O}$ ) (10.0 eq.) was added to a solution of the appropriate oxoacetate (1.0 eq.) in THF $(0.3 \mathrm{M})$ at $0{ }^{\circ} \mathrm{C}$. The reaction was stirred for $1 \mathrm{~h}$ at room temperature and then quenched with water. The aqueous phase was extracted with EtOAc. The combined organic phases were dried over $\mathrm{MgSO}_{4}$, filtered and the solvent was removed under reduced pressure.

\section{General procedure C: Perkin condensation}

$\mathrm{KO} t \mathrm{Bu}$ (1 $\mathrm{M}$ in THF) (1.2 eq.) was added to a solution of the appropriate amide (1.0 eq.) in THF $(0.2 \mathrm{M})$ at $0{ }^{\circ} \mathrm{C}$. After $90 \mathrm{~min}$ stirring at $0{ }^{\circ} \mathrm{C}$ the appropriate ester $\left(1.0\right.$ eq.) was added at $0{ }^{\circ} \mathrm{C}$ and stirred overnight at room temperature. The reaction was quenched with $1 \mathrm{M} \mathrm{HCl}$ and diluted with EtOAc. The organic phase was washed three times with water and one time with brine. The organic phase was dried over $\mathrm{MgSO}_{4}$, filtered and the solvent was removed under reduced pressure.

\section{2-(5-Chloro-2-methylthiophen-3-yl)-2-oxoacetamide (23)}

Compound 23 was prepared from 28 (800 $\mathrm{mg}, 3.66 \mathrm{mmol}$ ) according to general procedure B. The amide 23 (640 $\mathrm{mg}, 85 \%)$ was obtained as light yellow solid and used without further purification. m.p.: $183^{\circ} \mathrm{C}$; IR (neat) $\nu_{\max }: 3446,3252,1996,1670$, 1618, 1296, 1221, 1153; ${ }^{1} \mathrm{H}-\mathrm{NMR}\left(300 \mathrm{MHz}, \mathrm{DMSO}-d_{6}\right): \delta=2.64$ $\left(\mathrm{s}, 3 \mathrm{H}\right.$, thiophene- $\left.\mathrm{CH}_{3}\right), 7.49(\mathrm{~s}, 1 \mathrm{H}$, thiophene- $H), 7.94(\mathrm{bs}, 1 \mathrm{H}$, $\mathrm{NH}$ ), 8.25 (bs, $1 \mathrm{H}, \mathrm{N} H$ ); ${ }^{13} \mathrm{C}-\mathrm{NMR}$ (75 MHz, DMSO- $d_{6}$ ): $\delta=15.2$ $(+), 123.9$ (q), 128.4 (+), 131.4 (q), 150.9 (q), 166.1 (q), 184.2 (q); HRMS (ESI): calcd for $\mathrm{C}_{7} \mathrm{H}_{10} \mathrm{ClN}_{2} \mathrm{O}_{2} \mathrm{~S}\left(\mathrm{M}+\mathrm{NH}_{4}\right)^{+}$221.0146; found 221.0144.

\section{3,4-Bis(5-chloro-2-methylthiophen-3-yl)-1H-pyrrole-2,5-dione} (24)

Compound 24 was prepared from amide 23 (600 mg, 2.95 $\mathrm{mmol})$ and ester 22 (720 mg, $3.54 \mathrm{mmol})$ according to general procedure C. Purification by automated flash column chromatography (heptane/EtOAc: 5/1) yielded 24 (660 mg, 63\%) as orange solid. $R_{\mathrm{f}}$ : 0.18 (heptane/EtOAc: 5/1); m.p.: $237{ }^{\circ} \mathrm{C}$; IR (neat) $\nu_{\text {max }}: 3381,2939,2818,1653,1437,1002 ;{ }^{1} \mathrm{H}-\mathrm{NMR}$ (400 MHz, DMSO- $\left.d_{6}\right): \delta=1.87\left(\mathrm{~s}, 6 \mathrm{H}\right.$, thiophene- $\left.\mathrm{CH}_{3}\right), 6.97(\mathrm{~s}, 2 \mathrm{H}$, thiophene- $H$ ), 11.25 (bs, $1 \mathrm{H}, \mathrm{NH}$ ); ${ }^{13} \mathrm{C}-\mathrm{NMR}$ (101 MHz, DMSO$\left.d_{6}\right): \delta=14.6(+), 125.0(\mathrm{q}), 127.0(\mathrm{q}), 128.4(+), 133.6(\mathrm{q}), 140.0$ (q), 171.4 (q); HRMS (ESI): calcd for $\mathrm{C}_{14} \mathrm{H}_{10} \mathrm{Cl}_{2} \mathrm{NO}_{2} \mathrm{~S}_{2}(\mathrm{M}+\mathrm{H})^{+}$ 357.9525 ; found 357.9523 .

\section{Methyl 2-(5-(4-(tert-butyl)phenyl)-2-methylthiophen-3-yl)} acetate (26)

Compound 26 was prepared from 22 (200 $\mathrm{mg}, 0.98 \mathrm{mmol}$ ) according to general procedure A. Purification by automated flash column chromatography (PE/EtOAc, 0-25\% EtOAc) yielded 26 (163 mg, 55\%) as yellow liquid. $R_{\mathrm{f}}$ : 0.63 (PE/EtOAc: 5/1); IR (neat) $\nu_{\max }: 2961,1736,1609,1520,1435,1364,1239,1018$, 825; ${ }^{1} \mathrm{H}-\mathrm{NMR}\left(300 \mathrm{MHz}, \mathrm{CDCl}_{3}\right): \delta=1.33(\mathrm{~s}, 9 \mathrm{H}, t \mathrm{Bu}), 2.41(\mathrm{~s}$, $3 \mathrm{H}$, thiophene- $\left.\mathrm{CH}_{3}\right), 3.55\left(\mathrm{~s}, 2 \mathrm{H}\right.$, thiophene- $\left.\mathrm{CH}_{2} \mathrm{C}(\mathrm{O}) \mathrm{OCH}_{3}\right)$, $3.71\left(\mathrm{~s}, 3 \mathrm{H}\right.$, thiophene- $\left.\mathrm{CH}_{2} \mathrm{C}(\mathrm{O}) \mathrm{OCH}_{3}\right), 7.08$ (s, 1H, thiopheneH), 7.32-7.40 (m, 2H, Ph), 7.43-7.52 (m, 2H, Ph); ${ }^{13} \mathrm{C}-\mathrm{NMR}(75$ $\left.\mathrm{MHz}, \mathrm{CDCl}_{3}\right): \delta=13.3(+), 31.3(+), 34.1(-), 34.6(\mathrm{q}), 52.1(+)$, $124.7(+), 125.2(+), 125.7(+), 130.1(\mathrm{q}), 131.6(\mathrm{q}), 134.8(\mathrm{q}), 140.1$ (q), 150.2 (q), 171.6 (q); HRMS (ESI): calcd for $\mathrm{C}_{18} \mathrm{H}_{23} \mathrm{O}_{2} \mathrm{~S}(\mathrm{M}+$ $\mathrm{H})^{+}$303.1413; found 303.1418.

\section{Methyl 2-(5-([1,1'-biphenyl]-3-yl)-2-methylthiophen-3-yl) acetate (27)}

Compound 27 was prepared from 22 (500 mg, $2.44 \mathrm{mmol}$ ) according to general procedure A. Purification by automated flash column chromatography (PE/EtOAc, 0-15\% EtOAc) yielded 27 (569 mg, 72\%) as yellow liquid. $R_{\mathrm{f}}$ : 0.50 (PE/EtOAc: 5/1); IR (neat) $\nu_{\text {max }}: 1707,1597,1449,1262,1174,755,696 ;{ }^{1} \mathrm{H}-\mathrm{NMR}$ $\left(300 \mathrm{MHz}_{\mathrm{CDCl}}\right): \delta=2.44\left(\mathrm{~s}, 3 \mathrm{H}\right.$, thiophene- $\left.\mathrm{CH}_{3}\right), 3.58(\mathrm{~s}$, $2 \mathrm{H}$, thiophene- $\left.\mathrm{CH}_{2} \mathrm{C}(\mathrm{O}) \mathrm{OCH}_{3}\right), 3.72\left(\mathrm{~s}, 3 \mathrm{H}\right.$, thiophene- $\mathrm{CH}_{2} \mathrm{C}(\mathrm{O})$ $\left.\mathrm{OCH}_{3}\right), 7.19(\mathrm{~s}, 1 \mathrm{H}$, thiophene- $H)$, 7.37-7.45 (m, 3H, Ph), 7.467.54 (m, 3H, Ph), 7.59-7.66 (m, 2H, Ph), 7.74-7.76 (m, 1H, Ph); ${ }^{13} \mathrm{C}-\mathrm{NMR}\left(75 \mathrm{MHz}, \mathrm{CDCl}_{3}\right): \delta=2.44\left(\mathrm{~s}, 3 \mathrm{H}\right.$, thiophene- $\left.\mathrm{CH}_{3}\right), 3.58$ (s, 2H, thiophene- $\left.\mathrm{CH}_{2} \mathrm{C}(\mathrm{O}) \mathrm{OCH}_{3}\right), 3.72(\mathrm{~s}, 3 \mathrm{H}$, thiophene$\left.\mathrm{CH}_{2} \mathrm{C}(\mathrm{O}) \mathrm{OCH}_{3}\right), 7.19(\mathrm{~s}, 1 \mathrm{H}$, thiophene- $H), 7.37-7.42(\mathrm{~m}, 1 \mathrm{H}$, $\mathrm{Ph}$ ), 7.43-7.47 (m, 3H, Ph), 7.48-7.54 (m, 2H, Ph), 7.60-7.65 (m, 2H, Ph), 7.74-7.76 (m, 1H, Ph); ${ }^{13} \mathrm{C}-\mathrm{NMR}\left(75 \mathrm{MHz}, \mathrm{CDCl}_{3}\right): \delta=$ $13.3(+), 34.1(-), 52.1(+), 124.3(+), 124.4(+), 125.3(+), 126.0(+)$, $127.2(+), 127.5(+), 128.8(+), 129.2(+), 130.3(\mathrm{q}), 134.8(\mathrm{q}), 135.5$ (q), 139.9 (q), 140.9 (q), 141.9 (q), 171.5 (q); HRMS (EI): calcd for $\mathrm{C}_{20} \mathrm{H}_{18} \mathrm{O}_{2} \mathrm{~S}\left(\mathrm{M}^{+\bullet}\right)$ 322.1028; found 322.1032.

\section{Methyl 2-(5-(4-(tert-butyl)phenyl)-2-methylthiophen-3-yl)-2- oxoacetate (30)}

Compound 30 was prepared from 28 (500 $\mathrm{mg}, 2.29 \mathrm{mmol}$ ) according to general procedure A. Purification by automated 
flash column chromatography (PE/EtOAc, 0-15\% EtOAc) yielded 30 (350 mg, 48\%) as dark yellow liquid. $R_{\mathrm{f}}$ : 0.67 (PE/EtOAc: $5 / 1$ ); IR (neat) $\nu_{\text {max }}: 2961,2866,1732,1676,1456,1191,1127$, 993, 753; ${ }^{1} \mathrm{H}-\mathrm{NMR}\left(300 \mathrm{MHz}, \mathrm{CDCl}_{3}\right): \delta=1.34(\mathrm{~s}, 9 \mathrm{H}, t \mathrm{Bu}), 2.78$ $\left(\mathrm{s}, 3 \mathrm{H}\right.$, thiophene- $\left.\mathrm{CH}_{3}\right), 3.96\left(\mathrm{~s}, 3 \mathrm{H}\right.$, thiophene- $\left.\mathrm{CH}_{2} \mathrm{C}(\mathrm{O}) \mathrm{OCH}_{3}\right)$, 7.39-7.44 (m, 2H, Ph), 7.46-7.51 (m, 2H, Ph), 7.63 (s, 1H, thiophene- $H$ ); ${ }^{13} \mathrm{C}-\mathrm{NMR}\left(75 \mathrm{MHz}, \mathrm{CDCl}_{3}\right): \delta=16.3(+), 31.3(+), 34.7$ (q), $52.8(+), 124.2(+), 125.6(+), 126.0(+), 130.3(\mathrm{q}), 132.2(\mathrm{q})$, 140.5 (q), 151.3 (q), 153.5 (q), 164.1 (q), 180.2 (q); HRMS (EI): calcd for $\mathrm{C}_{18} \mathrm{H}_{20} \mathrm{O}_{3} \mathrm{~S}\left(\mathrm{M}^{+\bullet}\right)$ 316.1133; found 316.1139.

\section{Methyl 2-(5-([1,1'-biphenyl]-3-yl)-2-methylthiophen-3-yl)-2- oxoacetate (31)}

Compound 31 was prepared from 28 (500 $\mathrm{mg}, 2.29 \mathrm{mmol}$ ) according to general procedure A. Purification by automated flash column chromatography (PE/EtOAc, 0-15\% EtOAc) yielded 31 (537 mg, 70\%) as yellow oil. $R_{\mathrm{f}}$ : 0.40 (PE/EtOAc: 5/1); IR (neat) $\nu_{\text {max }}: 3028,1724,1668,1598,1464,1197,1132,1000,748$, 695; ${ }^{1} \mathrm{H}-\mathrm{NMR}\left(300 \mathrm{MHz}, \mathrm{CDCl}_{3}\right): \delta=2.81$ (s, 3H, thiophene$\left.\mathrm{CH}_{3}\right), 3.98\left(\mathrm{~s}, 3 \mathrm{H}\right.$, thiophene- $\left.\mathrm{CH}_{2} \mathrm{C}(\mathrm{O}) \mathrm{OCH}_{3}\right), 7.36-7.43(\mathrm{~m}, 1 \mathrm{H}$, $\mathrm{Ph}$ ), 7.45-7.49 (m, 2H, Ph), 7.50-7.57 (m, 3H, Ph), 7.60-7.65 (m, 2H, 4-H, Ph), 7.73-7.76 (m, 2H, Ph, thiophene- $H$ ); ${ }^{13} \mathrm{C}-\mathrm{NMR}$ (75 $\left.\mathrm{MHz}, \mathrm{CDCl}_{3}\right): \delta=16.4(+), 52.9(+), 124.6(+), 124.7(+), 124.8(+)$, $126.9(+), 127.2(+), 127.7(+), 128.9(+), 129.5(+), 132.3(\mathrm{q}), 133.5$ (q), 140.2 (q), 140.6 (q), 142.2 (q), 154.0 (q), 164.0 (q), 180.1 (q); HRMS (ESI): calcd for $\mathrm{C}_{20} \mathrm{H}_{15} \mathrm{O}_{2} \mathrm{~S}\left(\mathrm{M}+\mathrm{H}^{+}\right)-\left(\mathrm{H}_{2} \mathrm{O}\right) 319.0787$; found 319.0787 .

\section{2-(5-(4-(tert-Butyl)phenyl)-2-methylthiophen-3-yl)-2- oxoacetamide (33)}

Compound 33 was prepared from 30 (312 mg, $0.99 \mathrm{mmol}$ ) according to general procedure B. The amide 33 (250 $\mathrm{mg}, 84 \%$ ) was obtained as light yellow solid and used without further purification. m.p.: $202{ }^{\circ} \mathrm{C}$; IR (neat) $\nu_{\text {max }}: 3395,2955,1712,1651$, 1454, 1191, 575; ${ }^{1} \mathrm{H}-\mathrm{NMR}$ (300 MHz, DMSO- $\left.d_{6}\right): \delta=1.29(\mathrm{~s}, 9 \mathrm{H}$, $t \mathrm{Bu}), 2.71\left(\mathrm{~s}, 3 \mathrm{H}\right.$, thiophene- $\left.\mathrm{CH}_{3}\right), 7.36-7.48(\mathrm{~m}, 2 \mathrm{H}, \mathrm{Ph}), 7.51-$ $7.59(\mathrm{~m}, 2 \mathrm{H}, \mathrm{Ph}), 7.74(\mathrm{~s}, 1 \mathrm{H}$, thiophene- $H), 7.91(\mathrm{~s}, 1 \mathrm{H}, \mathrm{NH})$, $8.26(\mathrm{~s}, 1 \mathrm{H}, \mathrm{N} H) ;{ }^{13} \mathrm{C}-\mathrm{NMR}\left(75 \mathrm{MHz}, \mathrm{DMSO}-d_{6}\right): \delta=15.4(+), 30.9$ $(+), 34.3(\mathrm{q}), 124.4(+), 125.0(+), 126.0(+), 129.7(\mathrm{q}), 133.0(\mathrm{q})$, 138.9 (q), 150.5 (q), 150.6 (q), 167.0 (q), 185.5 (q); HRMS (ESI): calcd for $\mathrm{C}_{17} \mathrm{H}_{20} \mathrm{NO}_{2} \mathrm{~S}\left(\mathrm{M}+\mathrm{H}^{+}\right)$303.1240; found 303.1240.

\section{2-(5-([1,1'-Biphenyl]-3-yl)-2-methylthiophen-3-yl)-2- oxoacetamide (34)}

Compound 34 was prepared from 31 (250 $\mathrm{mg}, 0.74 \mathrm{mmol}$ ) according to general procedure B. The amide 34 (224 mg, 94\%) was obtained as light yellow solid and used without further purification. m.p.: $151^{\circ} \mathrm{C}$; IR (neat) $\nu_{\text {max }}: 3393,3302,3184,1721$, 1652, 1597, 1456, 1352, 1196, 747, 689, 608; ${ }^{1} \mathrm{H}-\mathrm{NMR}(300 \mathrm{MHz}$, DMSO- $\left.d_{6}\right): \delta=2.73\left(\mathrm{~s}, 3 \mathrm{H}\right.$, thiophene- $\left.\mathrm{CH}_{3}\right), 7.39-7.44(\mathrm{~m}, 1 \mathrm{H}$, $\mathrm{Ph}$ ), 7.47-7.56 (m, 3H, Ph), 7.58-7.66 (m, 2H, Ph), 7.71-7.75 (m, 2H, Ph), 7.83-7.85 (m, 1H, NH), 7.93 (bs, 2H, Ph, thiophene- $H$ ), 8.28 (bs, $1 \mathrm{H}, \mathrm{NH}) ;{ }^{13} \mathrm{C}-\mathrm{NMR}\left(75 \mathrm{MHz}, \mathrm{DMSO}-d_{6}\right): \delta=15.4(+)$, 123.4 (+), $124.4(+), 125.5(+), 126.4(+), 126.8(+), 127.7(+), 128.9$ $(+), 129.9(+), 133.1(\mathrm{q}), 133.2(\mathrm{q}), 138.7(\mathrm{q}), 139.5(\mathrm{q}), 141.1(\mathrm{q})$,
151.1 (q), 166.9 (q), 185.6 (q); HRMS (ESI): calcd for $\mathrm{C}_{19} \mathrm{H}_{16} \mathrm{NO}_{2} \mathrm{~S}$ $\left(\mathrm{M}+\mathrm{H}^{+}\right)$322.0896; found 322.0893.

\section{3-(5-(4-(tert-Butyl)phenyl)-2-methylthiophen-3-yl)-4-(5-chloro- 2-methylthiophen-3-yl)-1H-pyrrole-2,5-dione (35)}

Compound 35 was prepared from amide 23 (100 mg, 0.49 $\mathrm{mmol}$ ) and ester 26 (178 $\mathrm{mg}, 0.59 \mathrm{mmol}$ ) according to general procedure C. Purification by automated flash column chromatography (PE/EtOAc, 0-25\% EtOAc) yielded 35 (62 mg, 28\%) as dark green solid. $R_{\mathrm{f}}$ : 0.47 (PE/EtOAc: $\left.5 / 1\right)$; m.p.: $145{ }^{\circ} \mathrm{C}$; IR (neat) $\nu_{\max }: 3055,2961,1707,1459,1338,1181,1018,987,825 ;{ }^{1} \mathrm{H}-$ NMR (300 MHz, DMSO- $\left.d_{6}\right): \delta=1.28(\mathrm{~s}, 9 \mathrm{H}, t \mathrm{Bu}), 1.88(\mathrm{~s}, 3 \mathrm{H}$, thiophene- $\left.\mathrm{CH}_{3}\right), 1.99\left(\mathrm{~s}, 3 \mathrm{H}\right.$, thiophene- $\left.\mathrm{CH}_{3}\right), 7.02(\mathrm{~s}, 1 \mathrm{H}$, thiophene- $H$ ), $7.28(\mathrm{~s}, 1 \mathrm{H}$, thiophene- $H), 7.40-7.49(\mathrm{~m}, 4 \mathrm{H}, \mathrm{Ph})$, 11.27 (s, $1 \mathrm{H}, \mathrm{N} H)$; ${ }^{13} \mathrm{C}-\mathrm{NMR}\left(75 \mathrm{MHz}, \mathrm{DMSO}-d_{6}\right): \delta=14.0(+)$, $14.2(+), 30.9(+), 34.2(\mathrm{q}), 124.0(+), 124.3(\mathrm{q}), 124.7(+), 125.9(+)$, 126.7 (q), 127.8 (q), $127.9(+), 130.2(\mathrm{q}), 132.7$ (q), 134.1 (q), 139.3 (q), 139.5 (q), 139.8 (q), 150.3 (q), 171.0 (q), 171.1 (q); HRMS (ESI): calcd for $\mathrm{C}_{24} \mathrm{H}_{26} \mathrm{ClN}_{2} \mathrm{O}_{2} \mathrm{~S}_{2}\left(\mathrm{M}+\mathrm{NH}_{4}\right)^{+}$473.1119; found 473.1116 .

\section{3-(5-([1,1'-Biphenyl]-3-yl)-2-methylthiophen-3-yl)-4-(5-chloro- 2-methylthiophen-3-yl)-1H-pyrrole-2,5-dione (36)}

Compound 36 was prepared from amide 23 (100 mg, 0.49 $\mathrm{mmol}$ ) and ester 27 (160 mg, $0.49 \mathrm{mmol}$ ) according to general procedure C. Purification by automated flash column chromatography (PE/EtOAc, 0-25\% EtOAc) yielded 36 (63 mg, 27\%) as dark red solid. $R_{\mathrm{f}}: 0.16$ (PE/EtOAc: 5/1); m.p.: $137{ }^{\circ} \mathrm{C}$; IR (neat) $\nu_{\text {max }}: 2736,1705,1338,1022,1006,756,700 ;{ }^{1} \mathrm{H}-\mathrm{NMR}(300 \mathrm{MHz}$, DMSO- $\left.d_{6}\right): \delta=1.91\left(\mathrm{~s}, 3 \mathrm{H}\right.$, thiophene- $\left.\mathrm{CH}_{3}\right), 2.01(\mathrm{~s}, 3 \mathrm{H}$, thiophene- $\left.\mathrm{CH}_{3}\right), 7.03(\mathrm{~s}, 1 \mathrm{H}$, thiophene- $\mathrm{H}), 7.37-7.44(\mathrm{~m}, 1 \mathrm{H}, \mathrm{Ph})$, 7.46-7.56 (m, 5H, thiophene- $H, \mathrm{Ph}), 7.58-7.63(\mathrm{~m}, 1 \mathrm{H}, \mathrm{Ph})$, 7.69-7.75 (m, 2H, Ph), 7.77-7.79 (m, 1H, Ph), 11.30 (s, 1H, NH); ${ }^{13} \mathrm{C}-\mathrm{NMR}\left(75 \mathrm{MHz}, \mathrm{DMSO}-d_{6}\right): \delta=14.1(+), 14.2(+), 123.2(+)$, $124.1(+), 124.3(\mathrm{q}), 125.1(+), 126.1(+), 126.6(\mathrm{q}), 126.7(+), 127.7$ $(+), 127.9(+), 128.0(\mathrm{q}), 128.9(+), 129.8(+), 132.8(\mathrm{q}), 133.6(\mathrm{q})$, 134.0 (q), 139.4 (q), 139.5 (q), 139.6 (q), 140.2 (q), 141.1 (q), 171.0 (q), 171.1 (q); HRMS (ESI): calcd for $\mathrm{C}_{26} \mathrm{H}_{22} \mathrm{ClN}_{2} \mathrm{O}_{2} \mathrm{~S}_{2}\left(\mathrm{M}+\mathrm{NH}_{4}\right)^{+}$ 493.0806; found 493.0804 .

3-(5-([1,1'-Biphenyl]-3-yl)-2-methylthiophen-3-yl)-4-(5-(4-(tertbutyl)phenyl)-2-methylthiophen-3-yl)-1H-pyrrole-2,5-dione (37)

Compound 37 was prepared from amide 33 (260 mg, 0.86 $\mathrm{mmol}$ ) and ester 27 (277 $\mathrm{mg}, 0.86 \mathrm{mmol}$ ) according to general procedure C. Purification by automated flash column chromatography (PE/EtOAc, 0-15\% EtOAc) yielded 37 (155 mg, $33 \%$ ) as purple solid. $R_{\mathrm{f}}: 0.13$ (PE/EtOAc: 5/1); m.p.: $145{ }^{\circ} \mathrm{C}$; IR (neat) $\nu_{\max }: 2921,2851,1707,1334,831,756 ;{ }^{1} \mathrm{H}-\mathrm{NMR}(300$ MHz, DMSO- $\left.d_{6}\right): \delta=1.27(\mathrm{~s}, 9 \mathrm{H}, t \mathrm{Bu}), 2.02(\mathrm{~s}, 6 \mathrm{H}$, thiophene$\mathrm{CH}_{3}$, thiophene- $\left.\mathrm{CH}_{3}\right), 7.31(\mathrm{~s}, 1 \mathrm{H}$, thiophene- $H), 7.38-7.43(\mathrm{~m}$, $3 \mathrm{H}, \mathrm{Ph}), 7.44-7.50(\mathrm{~m}, 5 \mathrm{H}$, thiophene- $H, \mathrm{Ph}), 7.50-7.52(\mathrm{~m}, 1 \mathrm{H}$, $\mathrm{Ph})$, 7.54-7.56 (m, 1H, Ph), 7.58-7.61 (m, 1H, Ph), 7.68-7.71 (m, 2H, Ph), 7.76-7.78 (m, 1H, Ph), 11.27 (s, 1H, NH); ${ }^{13} \mathrm{C}-\mathrm{NMR}(75$ MHz, DMSO- $\left.d_{6}\right): \delta=14.3(+), 31.0(+), 34.3(\mathrm{q}), 123.2(+), 124.1$ $(+), 124.2(+), 124.8(+), 125.4(+), 126.0(+), 126.1(+), 126.8(+)$, 
$127.7(+), 128.2(\mathrm{q}), 128.3(\mathrm{q}), 128.9(+), 129.9(+), 130.3(\mathrm{q})$, 133.7 (q), 133.9 (q), 134.0 (q), 139.4 (q), 139.5 (q), 139.6 (q), 139.8 (q), 140.1 (q), 141.1 (q), 150.3 (q), 171.6 (q), 171.7 (q); HRMS (ESI): calcd for $\mathrm{C}_{36} \mathrm{H}_{32} \mathrm{NO}_{2} \mathrm{~S}_{2}(\mathrm{M}+\mathrm{H})^{+}$576.1879; found 576.1875 .

\section{Acknowledgements}

CF thanks the graduate research training group GRK 1910 for financial support.

\section{Notes and references}

1 J. Zhang, Q. Zou and H. Tian, Adv. Mater., 2013, 25, 378-399.

2 W. Szymański, J. M. Beierle, H. A. V. Kistemaker, W. A. Velema and B. L. Feringa, Chem. Rev., 2013, 113, 6114-6178.

3 D. Gust, J. Andreasson, U. Pischel, T. A. Moore and A. L. Moore, Chem. Commun., 2012, 48, 1947-1957.

4 S. Kawata and Y. Kawata, Chem. Rev., 2000, 100, 1777-1788. 5 C. C. Corredor, Z. L. Huang and K. D. Belfield, Adv. Mater., 2006, 18, 2910-2914.

6 V. A. Barachevsky, M. M. Krayushkin, V. V. Kyiko and E. P. Grebennikov, Phys. Status Solidi C, 2011, 8, 2841-2845.

7 D. Vomasta, C. Högner, N. R. Branda and B. König, Angew. Chem., Int. Ed., 2008, 47, 7644-7647.

8 D. Vomasta, A. Innocenti, B. König and C. T. Supuran, Bioorg. Med. Chem. Lett., 2009, 19, 1283-1286.

9 B. Reisinger, N. Kuzmanovic, P. Löffler, R. Merkl, B. König and R. Sterner, Angew. Chem., Int. Ed., 2014, 53, 595-598.

10 C. Falenczyk, M. Schiedel, B. Karaman, T. Rumpf, N. Kuzmanovic, M. Grøtli, W. Sippl, M. Jung and B. König, Chem. Sci., 2014, 5, 4794-4799.

11 M. Singer and A. Jäschke, J. Am. Chem. Soc., 2010, 132, 83728377.

12 S. Barrois and H.-A. Wagenknecht, Beilstein J. Org. Chem., 2012, 8, 905-914.

13 H. Cahová and A. Jäschke, Angew. Chem., Int. Ed., 2013, 52, 3186-3190.

14 M. R. Banghart, A. Mourot, D. L. Fortin, J. Z. Yao, R. H. Kramer and D. Trauner, Angew. Chem., Int. Ed., 2009, 48, 9097-9101.

15 T. Fehrentz, M. Schönberger and D. Trauner, Angew. Chem., Int. Ed., 2011, 50, 12156-12182.

16 I. Tochitsky, M. R. Banghart, A. Mourot, J. Z. Yao, B. Gaub, R. H. Kramer and D. Trauner, Nat. Chem., 2012, 4, 105-111.

17 A. Mourot, T. Fehrentz, Y. Le Feuvre, C. M. Smith, C. Herold, D. Dalkara, F. Nagy, D. Trauner and R. H. Kramer, Nat. Methods, 2012, 9, 396-402.

18 M. Schönberger and D. Trauner, Angew. Chem., Int. Ed., 2014, 53, 3264-3267.

19 M. Schönberger, M. Althaus, M. Fronius, W. Clauss and D. Trauner, Nat. Chem., 2014, 6, 712-719.

20 I. Tochitsky, A. Polosukhina, V. E. Degtyar, N. Gallerani, C. M. Smith, A. Friedman, R. N. Van Gelder, D. Trauner, D. Kaufer and R. H. Kramer, Neuron, 2014, 81, 800-813.
21 W. A. Velema, J. P. van der Berg, M. J. Hansen, W. Szymanski, A. J. M. Driessen and B. L. Feringa, Nat. Chem., 2013, 5, 924928.

22 O. Babii, S. Afonin, M. Berditsch, S. Reißer, P. K. Mykhailiuk, V. S. Kubyshkin, T. Steinbrecher, A. S. Ulrich and I. V. Komarov, Angew. Chem., Int. Ed., 2014, 53, 3392-3395.

23 U. Al-Atar, R. Fernandes, B. Johnsen, D. Baillie and N. R. Branda, J. Am. Chem. Soc., 2009, 131, 15966-15967.

24 W. A. Velema, W. Szymanski and B. L. Feringa, J. Am. Chem. Soc., 2014, 136, 2178-2191.

25 H. M. D. Bandara and S. C. Burdette, Chem. Soc. Rev., 2012, 41, 1809-1825.

26 G. Berkovic, V. Krongauz and V. Weiss, Chem. Rev., 2000, 100, 1741-1754.

27 Y. Yokoyama, Chem. Rev., 2000, 100, 1717-1740.

28 M. Irie, Chem. Rev., 2000, 100, 1685-1716.

29 H. Tian and S. Yang, Chem. Soc. Rev., 2004, 33, 85-97.

30 P. Raster, S. Weiss, G. Hilt and B. König, Synthesis, 2011, 2011, 905-908.

31 M. Dubernet, V. Caubert, J. Guillard and M.-C. ViaudMassuard, Tetrahedron, 2005, 61, 4585-4593.

32 S. V. Shorunov, M. M. Krayushkin, F. M. Stoyanovich and M. Irie, Russ. J. Org. Chem., 2006, 42, 1490-1497.

33 A. El Yahyaoui, G. Félix, A. Heynderickx, C. Moustrou and A. Samat, Tetrahedron, 2007, 63, 9482-9487.

34 M. M. Krayushkin, V. Z. Shirinyan, L. I. Belen'kii, A. A. Shimkin, A. Y. Martynkin and B. M. Uzhinov, Russ. J. Org. Chem., 2002, 38, 1335-1338.

35 M. Ohsumi, T. Fukaminato and M. Irie, Chem. Commun., 2005, 3921-3923.

36 M. T. Indelli, S. Carli, M. Ghirotti, C. Chiorboli, M. Ravaglia, M. Garavelli and F. Scandola, J. Am. Chem. Soc., 2008, 130, 7286-7299.

37 M. M. Faul, L. L. Winneroski and C. A. Krumrich, J. Org. Chem., 1998, 63, 6053-6058.

38 M. M. Faul, L. L. Winneroski and C. A. Krumrich, Tetrahedron Lett., 1999, 40, 1109-1112.

39 W. Steglich, Pure Appl. Chem., 1989, 81, 281-288.

40 T. Tamaoki and H. Nakano, Nat. Biotechnol., 1990, 8, 732735.

41 P. D. Davis, C. H. Hill, G. Lawton, J. S. Nixon, S. E. Wilkinson, S. A. Hurst, E. Keech and S. E. Turner, J. Med. Chem., 1992, 35, 177-184.

42 D. A. E. Cross, A. A. Culbert, K. A. Chalmers, L. Facci, S. D. Skaper and A. D. Reith, J. Neurochem., 2001, 77, 94-102.

43 L. Meijer, M. Flajolet and P. Greengard, Trends Pharmacol. Sci., 2004, 25, 471-480.

44 E. Khalifa, J. H. Bieri and M. Viscontini, Helv. Chim. Acta, 1973, 56, 2911-2919.

45 J. T. van Herpt, M. C. A. Stuart, W. R. Browne and B. L. Feringa, Chem. Eng. J., 2014, 20, 3077-3083.

46 K. Fujimoto, T. Maruyama, Y. Okada, T. Itou and M. Inouye, Tetrahedron, 2013, 69, 6170-6175.

47 K. Fuji, T. Kawabata and E. Fujita, Chem. Pharm. Bull., 1980, 28, 3662-3664.

48 J. W. Fisher and K. L. Trinkle, Tetrahedron Lett., 1994, 35, 2505-2508. 
49 Y. Goldberg and H. Alper, J. Org. Chem., 1993, 58, 3072-3075.

50 O. E. Levy, E. L. Madison, J. E. Semple, A. P. Tamiz and M. Weinhouse, US Pat., WO0214349 (A2), 2002.

51 C. Hamdouchi, US Pat., WO2013025424 (A1), 2013.

52 A. McKillop, B. P. Swann and E. C. Taylor, J. Am. Chem. Soc., 1973, 95, 3340-3343.
53 T. Yamaguchi and M. Irie, Chem. Lett., 2004, 33, 1398-1399. 54 T. Yamaguchi, K. Uchida and M. Irie, J. Am. Chem. Soc., 1997, 119, 6066-6071.

55 M. Irie and K. Sayo, J. Phys. Chem., 1992, 96, 7671-7674. 